\title{
REVISIÓN A 50 AÑOS DE LOS DAÑOS OCASIONADOS EN LA CIUDAD DE MÉXICO POR EL SISMO DEL 28 DE JULIO DE 1957 CON AYUDA DE INVESTIGACIONES RECIENTES Y SISTEMAS DE INFORMACIÓN GEOGRÁFICA
}

\author{
Vicente Orozco Narváez ${ }^{(1)}$ y Eduardo Reinoso Angulo ${ }^{(1)}$
}

\begin{abstract}
RESUMEN
En este trabajo se hace un recuento y revisión de los daños más sobresalientes ocasionados en varias estructuras de la Ciudad de México por el sismo del 28 de julio de 1957, conocido como el Temblor del Ángel, con el fin de conocer más sobre los efectos que influyeron en el comportamiento estructural y la presencia de daños. Para esto se realizó un análisis estadístico de varias estructuras considerando los efectos que pudieron intervenir como: tipo de sistema estructural, golpeteo, ubicación en esquina, forma en planta de la estructura, irregularidad en planta, número de niveles de la estructura, cercanía del valor del periodo del suelo y el periodo de la estructura, asentamientos diferenciales y tipo de cimentación. Con un sistema de información geográfica se visualizan los datos existentes y se realizaron mapas de intensidad sísmica de aceleración, velocidad y desplazamiento.
\end{abstract}

\begin{abstract}
With the purpose of identifying the main parameters that influenced the structural response and the presence of damage, a review of the damage caused to structures in Mexico City during the July $28^{\text {th }}, 1957$ earthquake, known as "Temblor del Ángel" is presented in this work. A statistical analysis of all collapsed and damaged structures was carried out, taking into account parameters such as: structural type, pounding, irregularity in plant (including buildings located in block's corners), number of stories, differential settlements and foundation type. Using a Geographical Information System we studied the existing building stock during 1957 together with the damaged structures and compare their behavior with maps of seismic intensity in terms of acceleration and displacements obtained with a scaled recent earthquake.
\end{abstract}

\section{INTRODUCCIÓN}

Hace cincuenta años la Ciudad de México se vio afectada el 28 de julio de 1957 por la ocurrencia de uno de los sismos más intensos en México durante el siglo XX (ICA, 1992). El sismo se originó en la costa del Pacífico pero los efectos en el centro de la ciudad de México fueron realmente sorprendentes debido al fenómeno de amplificación de la intensidad sísmica. En aquel momento las fuerzas sísmicas de diseño se obtenían a partir de un coeficiente sísmico de 0.025 para estructuras normales y de 0.05 para

Artículo recibido el 9 de octubre de 2006 y aprobado para su publicación el 27 de marzo. Se aceptarán comentarios y/o discusiones hasta cinco meses después de su publicación.

(1) Instituto de Ingeniería, UNAM. Circuito Escolar, Ciudad Universitaria, 04510 México, D.F. vorozcon@iingen.unam.mx,ere@pumas.iingen.unam.mx 
estructuras donde se acumulara un número importante de personas (ICA, 1985); estos coeficientes son claramente bajos para lo que hoy conocemos sobre la respuesta sísmica del valle de México.

La falta de registros del sismo impidió que se realizaran estudios rigurosos, sin embargo, destacan algunos trabajos relevantes. Figueroa (1957) y Rosenblueth (1958) hicieron observaciones sobre la amplificación en terreno blando, Marsal (1958) encontró que la mayoría de las estructuras reportadas con daño estaban desplantadas sobre terreno blando y Merino (1957) reportó que la zona de daño coincidía con la zona de mayores hundimientos de la ciudad. Zeevaert (1960) calculó a partir de una medición de la distorsión del primer piso de la torre Latinoamericana el coeficiente sísmico (0.036) en la base de la misma que sirvió como referencia para los coeficientes sísmicos recomendados a partir de ese momento. Lo anterior acentuó la necesidad de seguir investigando las propiedades del suelo de la ciudad de México y la colocación de instrumentos modernos de registro de aceleración en varios puntos de interés para tener datos completos durante sismos futuros.

A cincuenta años del primer sismo fuerte que motivo el desarrollo de la Ingeniería Sísmica Mexicana se presenta en este trabajo un estudio estadístico de varios edificios existentes durante el sismo de 1957 con el fin de conocer más sobre los efectos que influyeron en su comportamiento sísmico, como el golpeteo, tipo de sistema estructural, ubicación en esquina, número de niveles, periodo estructural, periodo del suelo, asentamientos diferenciales y tipo de cimentación, entre otros. Estos edificios se seleccionaron de los trabajos de Meli y col. (1985), Marsal (1958) y Excelsior (1957) tomando en cuenta que se tuviera la información de los daños que sufrieron durante el sismo y que estuvieran ubicados en la zona donde se presentaron la mayoría de los daños. Con fines prácticos, el área de estudio se limitó a la Delegación Cuauhtémoc. En la primera parte del trabajo se hace una clasificación de los edificios según el nivel de daño que tuvieron y se evalúa el comportamiento tomando en cuenta cada uno de los efectos mencionados. Se obtiene el periodo estructural de los edificios a partir de varias expresiones disponibles en la literatura y se utiliza un Sistema de Información Geográfica (SIG) para relacionar el nivel de daño con las características del suelo. Se simula el escenario de la intensidad que pudo tener el sismo de 1957 utilizando registros de otro sismo más reciente proveniente de la misma zona y con apoyo de un programa de cómputo, para finalmente obtener mapas de intensidad sísmica y su relación con los daños observados.

\section{DESCRIPCIÓN DEL SISMO DEL 28 DE JULIO DE 1957}

Figueroa (1957) reporta que el domingo 28 de julio de 1957 los sismógrafos ubicados en la estación de Tacubaya registraron a las 02 horas, 40 minutos, y 51 segundos un temblor sentido en el Distrito Federal proveniente del Pacífico frente a las costas de Guerrero. La magnitud calculada fue de 7.5 en la escala de Ritcher, con un valor de intensidad en el centro de la ciudad de México de VII en la escala de Mercalli modificada. El epicentro se calculó a $358 \mathrm{~km}$ al sur de Tacubaya con coordenadas $16^{\circ} 21^{\prime}$ N y $99^{\circ} 13^{\prime}$ W. En la fig. 1 se muestran el epicentro y las isosistas calculadas por Figueroa (1957).

Se estima que el número total de muertos en todo el país ascendió a 50 y el valor de los daños en lo que respecta a propiedad particular y gubernamental se calculó en 2 mil millones de pesos de esa época. Las poblaciones más afectadas además de la ciudad de México fueron San Marcos $(95 \%$ de edificios dañados), Chilpancingo ( $90 \%$ de edificios dañados), Chilapa ( $70 \%$ de edificios dañados), Huamuxtitlán (60\% de edificios dañados), Ayutla (con pérdidas estimadas de 360,000 pesos) y Tuxtla (60\% de edificios dañados), todas estas en el estado de Guerrero (Figueroa, 1957). 


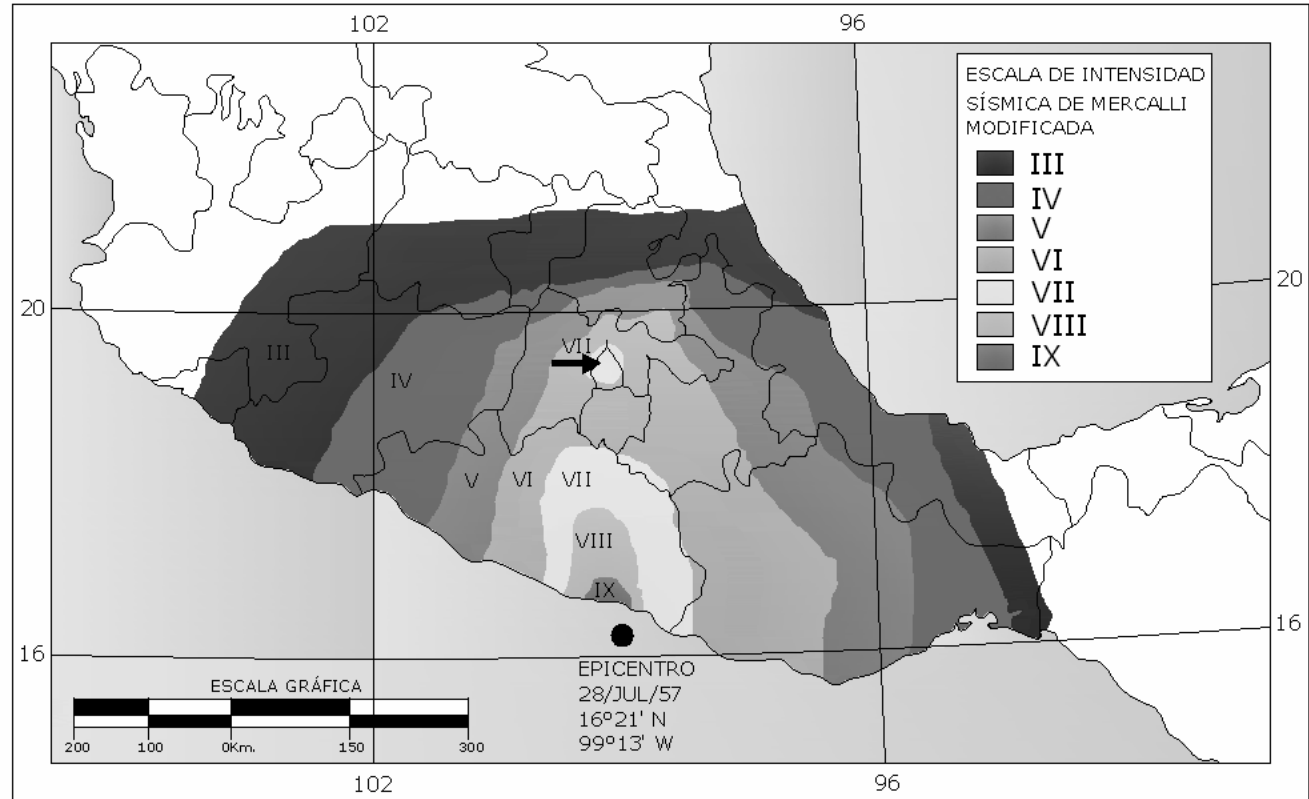

Figura 1. Curvas de isosistas y epicentro del sismo del 28/07/57.

\section{DESCRIPCIÓN DE LOS DAÑOS EN LA CIUDAD DE MÉXICO}

La mayoría de los daños ocurridos en la Ciudad de México se concentraron en la zona centro, en lo que hoy es la Delegación Cuauhtémoc. Según ICA (1992), se reportaron 39 muertos tan solo en la ciudad de México y alrededor de 1000 edificios con daños, incluyendo casos de bardas y estructuras con grietas y fisuras en acabados.

Este sismo es conocido como el "Temblor del Ángel”, debido a que la estatua que representa la Victoria Alada, comúnmente llamada El Ángel, que coronaba la Columna de la Independencia, cayó al suelo (fig. 2); ésta fue reconstruida casi en su totalidad y puesta en su lugar un año más tarde. La cabeza original no fue posible reconstruirla y está expuesta en el museo Casa de los Condes de Heras y Soto en el centro de la ciudad (Aguirre, 2003).
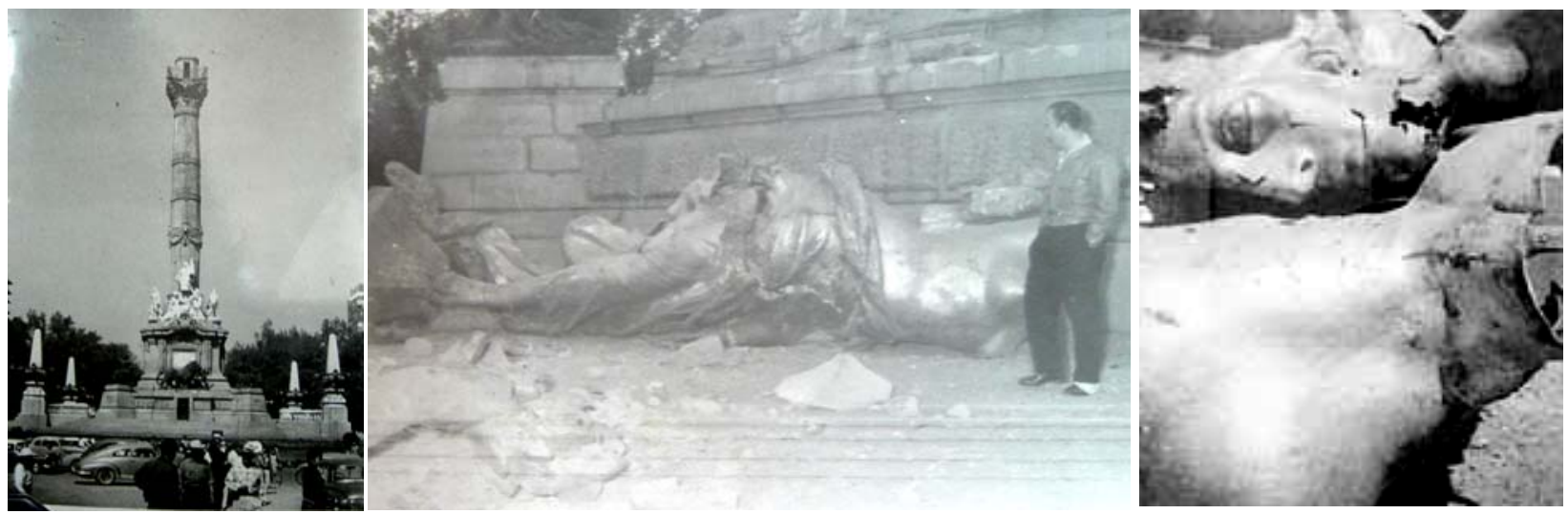

Figura 2. Columna y Ángel de la Independencia después del sismo del 28/07/57. Nótese en la primera imagen la ausencia de edificios altos detrás de la Columna de la Independencia. 
Las primeras noticias publicadas de los daños en la capital por Excelsior (1957) eran alarmantes y habían exagerado: "Casi no ha habido edificio que no haya sufrido desperfectos y numerosísimas bardas vinieron por tierra...", el encabezado del periódico del día 29 de junio de 1957 decía "35 Muertos, Cientos de Heridos y 100 Desplomes en la Capital".

Después de una comparación exhaustiva de lo publicado en aquella época por Excelsior (1957) y por los trabajos de Figueroa (1957), Marsal (1958) y Meli y col. (1985), encontramos que sólo hubo alrededor de cuatro colapsos totales y cinco parciales, por lo que concluimos que al decir desplomes no se referían a colapsos de edificios sino a bardas derribadas y a partes de techos que se vinieron abajo. De los trabajos anteriores se tomó la información de los edificios seleccionados y se elaboró la tabla 1 donde se muestra la ubicación, fecha de construcción, breve descripción de algunas de sus características estructurales y de los daños ocasionados por el sismo. Como se observa en la tabla 1, se clasificaron las estructuras según su sistema estructural en cinco grupos:

- Marcos de concreto reforzado (MC)

- Marcos de acero (MA)

- Construcción compuesta (CC), es decir, marcos de acero recubiertos por concreto

- Mampostería confinada (MC), estructuras que trasmiten la carga por medio de muros de tabique, con entrepisos y techo de concreto reforzado

- Naves industriales (NI), muros de tabique con confinamiento y cubierta formada por armaduras de acero y láminas ligeras

También se clasificaron las estructuras en cinco grupos según la magnitud del daño:

- Colapso total

- Colapso parcial. El edificio sufrió colapso de alguna zona del mismo, sea un piso intermedio, pisos superiores, colindancias o alguna esquina

- Daño grave. Daños en la mayoría de los elementos estructurales principales que pusieron en peligro la estabilidad de la estructura, no presenta colapso pero podría darse si no se corrigen los daños (en ocasiones se optó por demoler)

- Daño moderado. Grietas en elementos estructurales como trabes, losas y columnas. Deformaciones visibles o falla total de algunos elementos pero que no ponen en peligro de colapso al edificio

- Daño leve. Daño en elementos estructurales que no afectaron el funcionamiento del edificio manifestado en fisuras de poca magnitud.

La tabla 1 muestra todos los edificios que se reportaron con daños importantes en el Distrito Federal, pero sólo las estructuras que estaban dentro o cerca de los límites de la Delegación Cuauhtémoc se ubicaron utilizando un SIG en un mapa digital como se observa en la fig. 3. Las estructuras existentes en 1957 mostradas en la misma figura se obtuvieron de la base de datos del SIG proporcionada por la Subdirección de Catastro y Padrón Territorial del Gobierno del Distrito Federal (Quiroga y Reinoso, 2004). Esta base de datos no es muy exacta para 1957 por las remodelaciones que se han hecho a partir de esa fecha, pero nos puede dar una buena idea del universo de estructuras de esa época dentro de la delegación. 
Revisión a 50 años de los daños ocasionados en la Ciudad de México por el sismo del 28 de julio de 1957 con ...

Tabla 1. Descripción de estructuras dañadas por el sismo del 28/07/57.

\begin{tabular}{|c|c|c|c|c|c|c|c|c|c|c|c|c|c|c|c|c|c|}
\hline \multirow{3}{*}{ 总 } & \multirow{2}{*}{\multicolumn{2}{|c|}{ Dirección }} & \multirow{3}{*}{ 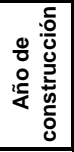 } & \multirow{3}{*}{ 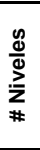 } & \multirow{3}{*}{ Uso } & \multirow{3}{*}{ 을 } & \multirow{3}{*}{ 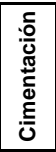 } & \multirow{3}{*}{ 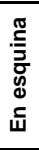 } & \multirow{3}{*}{ 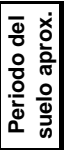 } & \multirow{3}{*}{ 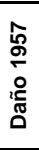 } & \multicolumn{2}{|l|}{ Daño estructural } & \multirow{3}{*}{ 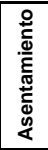 } & \multicolumn{4}{|c|}{\begin{tabular}{|l|} 
Daño no estructural \\
\end{tabular}} \\
\hline & & & & & & & & & & & \multirow[b]{2}{*}{ Descripción del daño en elementos } & \multirow{2}{*}{ 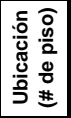 } & & \multirow{2}{*}{ 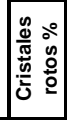 } & \multirow{2}{*}{ 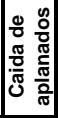 } & \multicolumn{2}{|c|}{\begin{tabular}{|c|c|} 
Destrucción \\
de muros
\end{tabular}} \\
\hline & Calle & \# & & & & & & & & & & & & & & $\frac{\bar{\pi}}{\frac{\pi}{2}}$ & 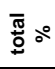 \\
\hline $57-05$ & $\begin{array}{l}\text { Aquiles } \\
\text { Serdán }\end{array}$ & 29 & 1950 & 13 & Oficinas & MC & PM & $\mathrm{Si}$ & 2.21 & $\mathrm{~L}$ & $\begin{array}{c}\text { Flexión en columnas, tensión } \\
\text { diagonal en trabes y tensión simple } \\
\text { en losas del cubo de escaleras }\end{array}$ & 2 al 4 & $\mathrm{Si}$ & 60 & Si & 70 & 10 \\
\hline $57-06$ & Insurgentes & 37 & 1947 & 16 & Oficinas & $\mathrm{MC}$ & PM & $\mathrm{Si}$ & 2.23 & $\mathrm{~L}$ & Tensión diagonal en trabes & 4 y 5 & $\mathrm{Si}$ & 5 & Si & 50 & --- \\
\hline $57-07$ & Balderas & 44 & 1941 & 6 & $\begin{array}{l}\text { Oficinas y } \\
\text { Escuela }\end{array}$ & MC & S & No & 2.18 & L & Tensión diagonal en trabes & 3 & Si & 10 & Si & 10 & --- \\
\hline $57-12$ & Chapultepec & 540 & 1953 & 8 & Oficinas & MC & S & No & 1.14 & L & Falla por flexión en columnas & 4 & No & 15 & --- & 30 & 40 \\
\hline $57-25$ & Gutemberg & 47 & 1949 & 11 & Vivienda & MC & PM & No & 1.11 & $\mathrm{~L}$ & $\begin{array}{c}\begin{array}{c}\text { Flexión en trabes. Daños en losa de } \\
\text { escalera, hubo necesidad de } \\
\text { apuntalar }\end{array} \\
\end{array}$ & 2 al 4 & No & 10 & Si & 40 & 40 \\
\hline $57-26$ & Havre & 7 & 1952 & 9 & Oficinas & $\mathrm{MC}$ & $\mathrm{CC}$ & No & 1.91 & $\mathrm{~L}$ & Tensión diagonal en trabes & 1,3 & $\mathrm{Si}$ & --- & $-\cdots$ & 100 & --- \\
\hline $57-41$ & Insurgentes & 428 & 1947 & 5 & Vivienda & $\mathrm{MC}$ & $\mathrm{S}$ & No & 1.17 & $\mathrm{~L}$ & Pandeo en columnas & 3 & No & --- & --- & 10 & --- \\
\hline $57-44$ & Insurgentes & 473 & 1942 & 8 & Oficinas & MC & PM & No & 1.1 & L & $\begin{array}{c}\text { Falla moderada en trabes por tensión } \\
\text { diagonal }\end{array}$ & 2 & No & 80 & $\mathrm{Si}$ & 20 & 40 \\
\hline $57-45$ & Insurgentes & 576 & 1950 & 13 & Vivienda & $\mathrm{MC}$ & PM & No & 1.06 & $\mathrm{~L}$ & Tensión diagonal en trabes & $1 \mathrm{al} 7$ & No & 5 & $\mathrm{Si}$ & 60 & --- \\
\hline $57-53$ & La Fragua & 4 & --- & 7 & Oficinas & MC & $\mathrm{CC}$ & No & 2.03 & $\mathrm{~L}$ & $\begin{array}{c}\text { Flexión en columnas, tensión } \\
\text { diagonal en trabes de lindero norte, } \\
\text { hubo golpeteo }\end{array}$ & 2 al 4 & No & 5 & SI & 20 & 20 \\
\hline $57-57$ & M. Ocampo & 257 & 1944 & 11 & Vivienda & MC & --- & $\mathrm{Si}$ & 1.15 & L & $\begin{array}{c}\text { Tensión, tensión diagonal y esfuerzo } \\
\text { cortante en trabes }\end{array}$ & 2 al 4 & No & 10 & $\mathrm{Si}$ & 50 & 50 \\
\hline $57-70$ & Reforma & 45 & 1950 & 19 & Oficinas & $\mathrm{CC}$ & $\mathrm{CC}$ & $\mathrm{Si}$ & 2.08 & $\mathrm{~L}$ & $\begin{array}{c}\text { Flexión en trabes de cubo de } \\
\text { elevadores }\end{array}$ & 6 al 9 & Si & 5 & Si & 60 & --- \\
\hline $57-75$ & Reforma & 104 & 1952 & 9 & Oficinas & $\mathrm{MC}$ & $\mathrm{PCP}$ & No & 2.17 & $\mathrm{~L}$ & \begin{tabular}{|c|} 
Tensión diagonal en trabes, tensión \\
simple en losas de cubo de \\
escaleras
\end{tabular} & 2 al 5 & Si & 50 & --- & 30 & --- \\
\hline $57-80$ & Reforma & 336 & 1948 & 7 & Oficinas & $\mathrm{MC}$ & $\mathrm{S}$ & No & 1.87 & $\mathrm{~L}$ & Tensión diagonal en trabes & $1 \mathrm{al} 3$ & No & 30 & $\mathrm{Si}$ & 30 & 10 \\
\hline $57-92$ & Sullivan & 199 & 1955 & 11 & Vivienda & MC & CC & $\mathrm{Si}$ & 1.28 & $\mathrm{~L}$ & $\begin{array}{c}\text { Grietas de tensión diagonal en trabes } \\
\text { cortas del techo del sótano }\end{array}$ & P.B. & No & 10 & Si & 20 & --- \\
\hline $57-15$ & \begin{tabular}{|l} 
Dr. \\
Barragán \\
\end{tabular} & 538 & 1940 & 3 & Vivienda & $\mathrm{MmC}$ & $S$ & No & 2.92 & M & \begin{tabular}{|c|}
$\begin{array}{c}\text { Esfuerzo cortante en muros y tensión } \\
\text { simple en losas por golpeteo }\end{array}$ \\
\end{tabular} & $\begin{array}{c}\text { P.B. y } \\
1\end{array}$ & $\mathrm{Si}$ & 5 & Si & 60 & 10 \\
\hline $57-50$ & Juárez & 76 & 1938 & 11 & Oficinas & MC & PM & $\mathrm{Si}$ & 2.17 & $M$ & $\begin{array}{c}\text { Tensión diagonal y esfuerzo cortante } \\
\text { en trabes, tensión simple en losas }\end{array}$ & $\begin{array}{l}\text { P.B. } \\
2 \text { al } 4\end{array}$ & Si & 30 & Si & 10 & --- \\
\hline $57-60$ & Morelos & 110 & 1945 & 13 & Oficinas & MC & PM & $\mathrm{Si}$ & 2.16 & M & $\begin{array}{c}\text { Flexión y tensión diagonal en } \\
\text { columnas y trabes respectivamente }\end{array}$ & $\begin{array}{l}6 \mathrm{al} \\
12 \\
\end{array}$ & Si & 10 & Si & 30 & 30 \\
\hline $57-65$ & Oaxaca & 50 & 1955 & 11 & Oficinas & MC & PCP & No & 1.75 & $M$ & $\begin{array}{l}\text { Falla total de pilotes de control, el } \\
\text { asentamiento diferencial fue de } 1 \mathrm{~m}\end{array}$ & P.B. & $\mathrm{Si}$ & --- & --- & 10 & --- \\
\hline 57-76 & Reforma & 122 & 1951 & 14 & Oficinas & $\mathrm{MC}$ & $\mathrm{PCP}$ & No & 2.19 & M & $\begin{array}{l}\text { Flexión en columnas y tensión en } \\
\text { trabes de cubo de escalera. Tensión } \\
\text { diagonal en trabes por golpeteo }\end{array}$ & $\left|\begin{array}{c}3 \text { al } 8 \\
y \\
2 \text { al } 6\end{array}\right|$ & No & 5 & $\mathrm{Si}$ & 5 & --- \\
\hline $57-83$ & Río Rhin & 82 & 1935 & 3 & Vivienda & $\mathrm{MmC}$ & $S$ & No & 1.69 & M & $\begin{array}{c}\text { Derrumbe parcial losas de balcones } \\
\text { de la zona posterior }\end{array}$ & 3 & No & --- & No & --- & --- \\
\hline $57-86$ & $\begin{array}{l}\begin{array}{l}\text { San Juan de } \\
\text { Letrán }\end{array} \\
\end{array}$ & 23 & 1936 & 7 & Cine & MC & S & No & 2.36 & $M$ & Flexión en columnas por golpeteo & 2 al 6 & No & 40 & Si & 10 & 90 \\
\hline $57-90$ & Soledad & 90 & 1930 & 3 & Comercio & $\mathrm{MmC}$ & S & $\mathrm{Si}$ & 2.54 & M & $\begin{array}{l}\text { Tensión simple en muros y losas. } \\
\text { Peligro de derrumbe en voladizos }\end{array}$ & $\begin{array}{l}\text { P.B. } \\
\text { al } 2\end{array}$ & Si & --- & Si & 10 & --- \\
\hline $57-93$ & Topógrafos & 7 & 1952 & 14 & Vivienda & MC & PC & $\mathrm{Si}$ & 0.93 & M & $\begin{array}{c}\text { Flexión en columnas, tensión en } \\
\text { losas y esfuerzo cortante en trabes }\end{array}$ & $\begin{array}{c}\text { al } \\
11 \\
\end{array}$ & No & 5 & Si & 70 & --- \\
\hline $57-C 5$ & --- & --- & --- & 15 & Oficinas & $\mathrm{CC}$ & PM & No & -- & M & $\begin{array}{c}\text { Grietas en recubrimientos de } \\
\text { estructura }\end{array}$ & --- & No & 10 & Si & 30 & 40 \\
\hline $57-C 7$ & --- & --- & 1956 & 9 & Vivienda & $\mathrm{MC}$ & PCP & $\mathrm{Si}$ & -- & $M$ & $\begin{array}{l}\text { Falla de un contraviento en P.B., } \\
\text { agritamiento columna que lo recibe }\end{array}$ & P.B. & No & --- & Si & 30 & --- \\
\hline 57-C10 & --- & --- & --- & 11 & Oficinas & MC & $\mathrm{CC}$ & No & -- & $M$ & $\begin{array}{l}\text { Trabes de colindancia se agrietaron, } \\
\text { varios elementos del cubo de } \\
\text { elevadores tuvieron daños severos. } \\
\text { Daños en fachada por golpeteo }\end{array}$ & --- & No & --- & Si & --- & --- \\
\hline $57-03$ & $\begin{array}{l}\text { Abraham } \\
\text { González }\end{array}$ & 3 & 1947 & 10 & Oficinas & MC & PM & $\mathrm{Si}$ & 2.16 & G & $\begin{array}{c}\text { Flexión columnas, tensión diagonal } \\
\text { en trabes y pandeo en losas }\end{array}$ & 2 al 4 & No & 20 & $\mathrm{Si}$ & 10 & 80 \\
\hline
\end{tabular}

Tipo de cimentación: S Superficial, CC Cajón de Cimentación, PCP Parcialmente compensada con cajón y pilotes, PC Pilotes de Concreto (fricción), PM Pilotes de Madera (punta)

Nivel de Daño: L Leve, M Moderado, G Grave, CP Colapso Parcial, CT Colapso Total

Sistemas Estructurales: MC Marcos de Concreto, MA Marcos de Acero, CC Construcción Compuesta, MmC Mampostería Confinada, NI Naves Industriales 
Tabla 1. Descripción de estructuras dañadas por el sismo del 28/07/57 (continuación).

\begin{tabular}{|c|c|c|c|c|c|c|c|c|c|c|c|c|c|c|c|c|c|}
\hline \multirow{3}{*}{ 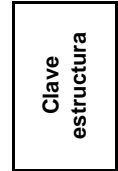 } & \multirow{2}{*}{\multicolumn{2}{|c|}{ Dirección }} & \multirow{3}{*}{ 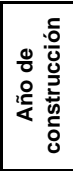 } & \multirow{3}{*}{ 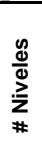 } & \multirow{3}{*}{ Uso } & \multirow{3}{*}{ 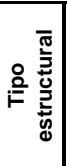 } & \multirow{3}{*}{ 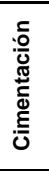 } & \multirow{3}{*}{ 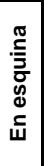 } & \multirow{3}{*}{ 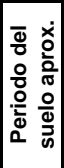 } & \multirow{3}{*}{ 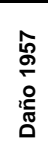 } & \multicolumn{2}{|l|}{ Daño estructural } & \multirow{3}{*}{ 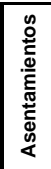 } & \multicolumn{4}{|c|}{ Daño no estructural } \\
\hline & & & & & & & & & & & \multirow[b]{2}{*}{ Descripción del daño en elementos } & \multirow{2}{*}{ 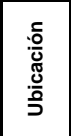 } & & \multirow[b]{2}{*}{$\underline{\underline{t}}$} & \multirow{2}{*}{ 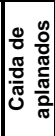 } & \multicolumn{2}{|c|}{\begin{tabular}{|c} 
Destrucciór \\
de muros
\end{tabular}} \\
\hline & Calle & $\#$ & & & & & & & & & & & & & & 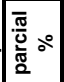 & $\frac{\circ}{\frac{\pi}{\pi}}$ \\
\hline $57-22$ & $\begin{array}{l}\text { Casco de } \\
\text { Sto. Tomas }\end{array}$ & -- & 1950 & 4 & Escuela & MC & $\mathrm{S}$ & No & 0.7 & G & $\begin{array}{c}\text { Flexión en columnas y tensión } \\
\text { diagonal en trabes en crujías } \\
\text { alejadas de zona esquina }\end{array}$ & P.B. & No & --- & No & 30 & --- \\
\hline $57-33$ & $\begin{array}{l}\text { Aquiles } \\
\text { Serdán }\end{array}$ & 29 & 1951 & 9 & Oficinas & MC & $\mathrm{PC}$ & $\mathrm{Si}$ & 2.04 & G & \begin{tabular}{|c|} 
Tensión diagonal en trabes. Falla de \\
control de pilotes
\end{tabular} & 2 y 3 & $\mathrm{Si}$ & 25 & Si & 40 & 20 \\
\hline $57-37$ & Insurgentes & 263 & 1946 & 9 & Oficinas & MC & PM & $\mathrm{Si}$ & 1.78 & G & $\begin{array}{c}\text { Tensión diagonal en trabes, flexión } \\
\text { en columnas }\end{array}$ & 5 al 8 & Si & 5 & Si & 30 & --- \\
\hline $57-38$ & Insurgentes & 368 & 1948 & 5 & Banco & $\mathrm{MC}$ & $\mathrm{S}$ & $\mathrm{Si}$ & 1.44 & $\mathrm{G}$ & Flexión en columnas & P.B. & No & --- & No & --- & --- \\
\hline $57-55$ & Lecumberri & 63 & 1946 & 2 & Cine & $\mathrm{NI}$ & $\mathrm{S}$ & No & 2.62 & $\mathrm{G}$ & Volcamiento de armaduras de techo & 2 & No & --- & No & 10 & 50 \\
\hline $57-69$ & Reforma & 35 & 1956 & 14 & Oficinas & MC & $\mathrm{CC}$ & No & 2.06 & G & $\begin{array}{c}\text { Compresión y tensión diagonal en } \\
\text { trabes, flexión en columnas }\end{array}$ & 2 al 5 & Si & 5 & Si & 10 & --- \\
\hline $57-74$ & Reforma & 77 & 1943 & 14 & Oficinas & MA & PM & $\mathrm{Si}$ & 2.06 & $\mathrm{G}$ & Flexión en columnas & $1 \mathrm{al} 3$ & No & 15 & Si & 30 & 50 \\
\hline $57-98$ & Villalongin & --- & --- & 5 & Oficinas & MC & PM & No & 1.28 & G & Tensión diagonal en trabes & $\begin{array}{l}\text { P.B. } \\
\text { al } 4\end{array}$ & Si & 30 & $\mathrm{Si}$ & 20 & 25 \\
\hline 57-Corcu & Reforma & 1 & 1934 & 16 & Oficinas & MC & --- & Si & 2.05 & G & $\begin{array}{c}\text { Sin datos exactos. El edificio fue } \\
\text { demolido después del sismo }\end{array}$ & --- & No & 10 & Si & 10 & 10 \\
\hline $57-C 4$ & --- & --- & 1957 & 8 & $\begin{array}{l}\text { Comercio } \\
\text { y oficina }\end{array}$ & MC & $\mathrm{CC}$ & No & -- & G & $\begin{array}{l}\text { Fractura en parte superior de varias } \\
\text { columnas, tensión diagonal y corte } \\
\text { en vigas y falla total en algunas }\end{array}$ & $\begin{array}{l}\text { P.B. } \\
\text { al } 8\end{array}$ & No & 50 & Si & --- & 20 \\
\hline $57-\mathrm{C} 6$ & --- & --- & --- & 16 & --- & MC & PM & No & -- & G & $\begin{array}{c}6 \text { columnas fallaron casi totalmente y } \\
\text { las restantes estan agrietadas. } \\
\text { Trabes con tensión diagonal }\end{array}$ & $\begin{array}{c}5,8 y \\
9\end{array}$ & Si & 50 & Si & 60 & --- \\
\hline $57-\mathrm{C9}$ & --- & --- & --- & 7 & --- & MC & $\mathrm{CC}$ & $\mathrm{Si}$ & -- & G & $\begin{array}{l}\text { Grietas en union de columnas } \\
\text { perimetrales con trabes. Tensión } \\
\text { diagonal en vigas principales y } \\
\text { escaleras a punto de derrumbe }\end{array}$ & 1 al 4 & No & 40 & Si & 40 & --- \\
\hline $57-46$ & Insurgentes & 1338 & 1955 & 1 & $\begin{array}{l}\text { Bodegas } \\
\text { Oficinas }\end{array}$ & $\mathrm{NI}$ & $\mathrm{S}$ & No & 1.42 & $\mathrm{CP}$ & $\begin{array}{l}\text { Colapso de oficinas. Flexión grave } \\
\text { en columnas, daño en mensulas }\end{array}$ & 1 & Si & 100 & Si & --- & 100 \\
\hline 57-Gera & Geranio & - & --- & 5 & Oficinas & -- & --- & Si & 1.46 & $\mathrm{CP}$ & Colapso parcial de pisos superiores & 5 & \begin{tabular}{|l|l}
--- \\
\end{tabular} & 20 & $\mathrm{Si}$ & 15 & 30 \\
\hline 57-Enca & $\begin{array}{l}\text { Serapio } \\
\text { Rendon }\end{array}$ & - & -- & 1 & Cine & $\mathrm{NI}$ & --- & No & 1.63 & $\mathrm{CP}$ & Derrumbe total del techo & 1 & No & --- & Si & 100 & --- \\
\hline 57-Merce & $\begin{array}{l}\text { Santa } \\
\text { Escuela }\end{array}$ & - & 1957 & 1 & Comercio & $\mathrm{NI}$ & $\mathrm{CC}$ & No & 2.96 & $\mathrm{CP}$ & $\begin{array}{c}6 \text { cascarones colapsaron y los } 88 \\
\text { restantes fallaron por flexión en el } \\
\text { arranque de las columnas }\end{array}$ & 1 & No & --- & No & --- & --- \\
\hline 57-Cant & Insurgentes & 337 & $\cdots$ & 5 & Oficina & $\mathrm{MC}$ & --- & No & 1.42 & $\mathrm{CP}$ & Colapso pisos superiores & 2 al 5 & --- & 85 & $\mathrm{Si}$ & 5 & 80 \\
\hline 57-Fron & $\begin{array}{l}\text { Frontera } \\
\text { esq. Alvaro } \\
\text { Obregón }\end{array}$ & --- & 1957 & 5 & Oficina & MC & --- & $\mathrm{Si}$ & 2.27 & CT & Colapso total & todos & --- & 100 & Si & --- & 100 \\
\hline 57-IPN & $\begin{array}{l}\text { Casco de } \\
\text { Sto. Tomas }\end{array}$ & --- & 1950 & 4 & Escuela & MC & $\mathrm{S}$ & No & 0.97 & CT & Colapso total & todos & --- & 100 & Si & --- & 100 \\
\hline 57-S/Mu & $\begin{array}{l}\text { C. Romero } \\
\text { de Terreros }\end{array}$ & 52 & 1957 & 2 & Vivienda & $\mathrm{MmC}$ & $\mathrm{S}$ & No & 1.25 & CT & $\begin{array}{c}\text { Colapso total. No tenía muros en } \\
\text { sentido NS }\end{array}$ & todos & --- & 100 & $\mathrm{Si}$ & -- & 100 \\
\hline
\end{tabular}

\section{Colapsos totales}

A continuación se describen brevemente los casos de colapso total mostrados en la tabla 1:

- Escuela Superior de Ingeniería y Arquitectura del IPN (57-IPN, fig. 3 y tabla 1). Estructura de marcos de concreto reforzado de 4 pisos y planta baja sin ningún muro. No se registró ninguna pérdida humana pero sí el sismo hubiera ocurrido en horario de clases habría causado la muerte de cientos de estudiantes (fig. 4a).

- Edificio en la esquina de las calles de Frontera y Álvaro Obregón (57-Fron, fig. 3 y tabla 1). Estructura de marcos de concreto reforzado de 5 pisos, destinado a vivienda; en este colapso se reportaron 31 muertos (fig. 4b). Fue el caso más trágico, "La construcción se desplomó sobre sus propios cimientos, como un gigantesco acordeón que se cierra verticalmente, de tal manera que los escombros no alcanzaron a rebasar la banqueta" (Excelsior, 1957). 


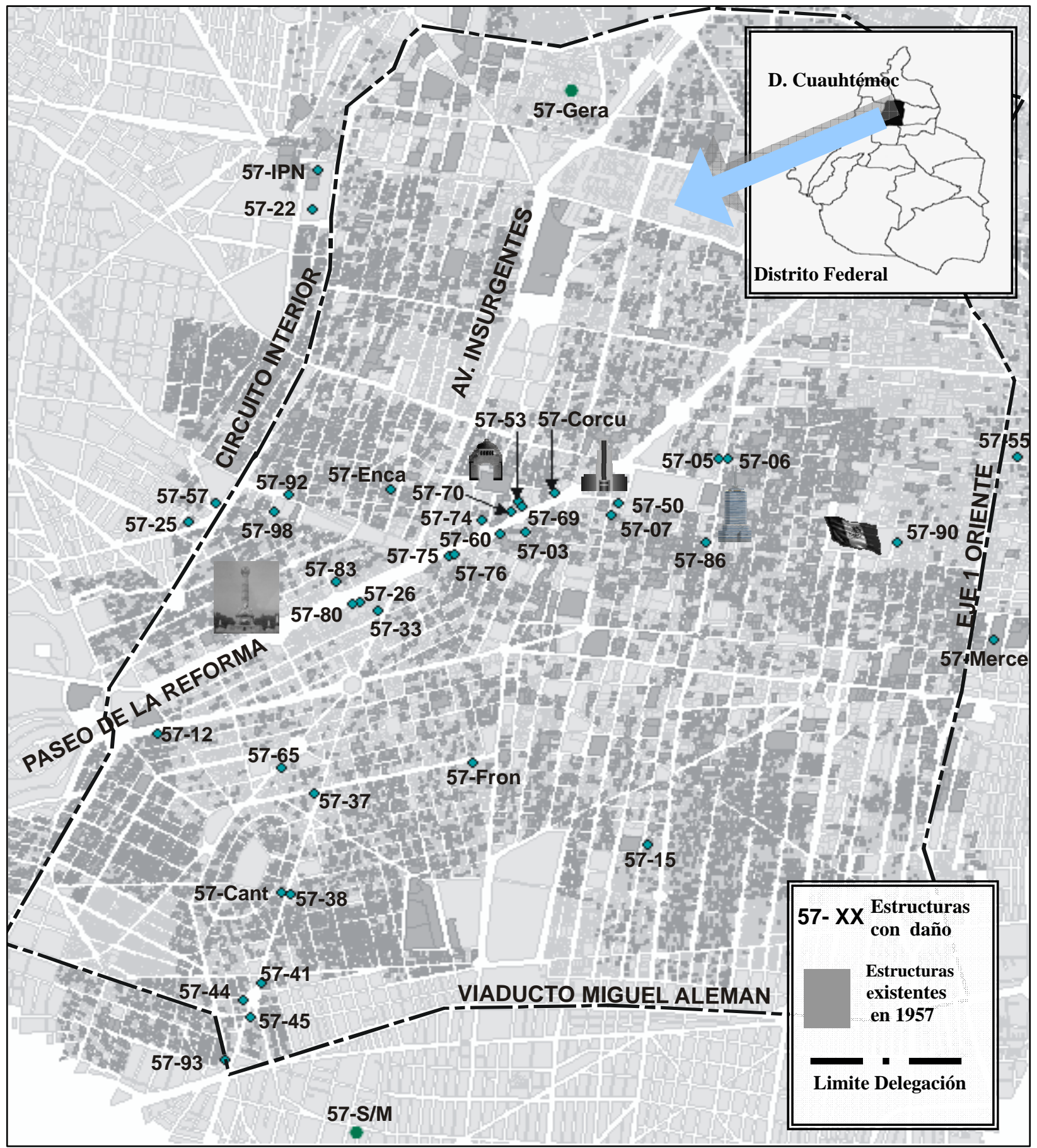

Figura 3. Ubicación de estructuras con daños ocasionados por el sismo del 28/07/57 en un Sistema de Información Geográfica (SIG). Se muestra esquemáticamente la ubicación de algunas referencias de la Delegación Cuauhtémoc. En gris oscuro se muestran los edificios que estaban en 1957 y no han sido modificados o sustituidos. 


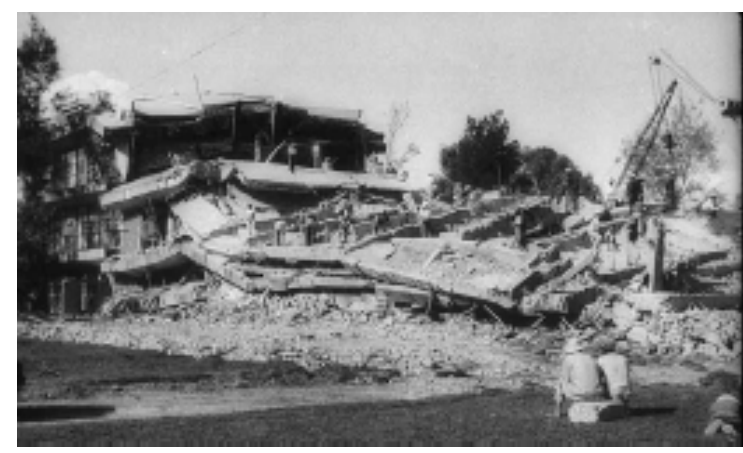

a)

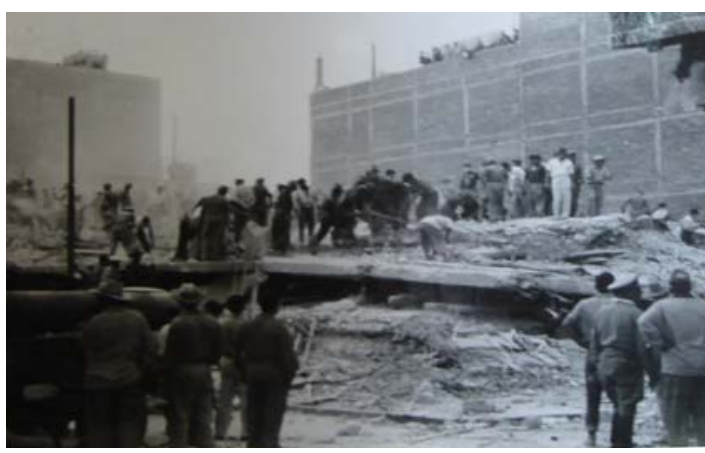

b)

Figura 4. Colapsos totales: a) Escuela Superior de Ingeniería y Arquitectura, IPN y b) Edificio en esquina de las calles de Frontera y Álvaro Obregón.

- Casa en la cerrada de Romero de Terreros \# 52 (57-S/M, fig. 3 y tabla 1). Estructura que estaba en construcción con muros de mampostería unidos con dalas y castillos de concreto reforzado de 2 pisos. En la fig. 5 se muestra el plano de planta baja de la casa donde se observa deficiencia de muros y castillos en la dirección Norte-Sur (Marsal, 1958); se reportó que la estructura se desplazó en esa dirección. En esa época el RCDF no exigía la revisión de estructuras con altura menor de $16 \mathrm{~m}$.

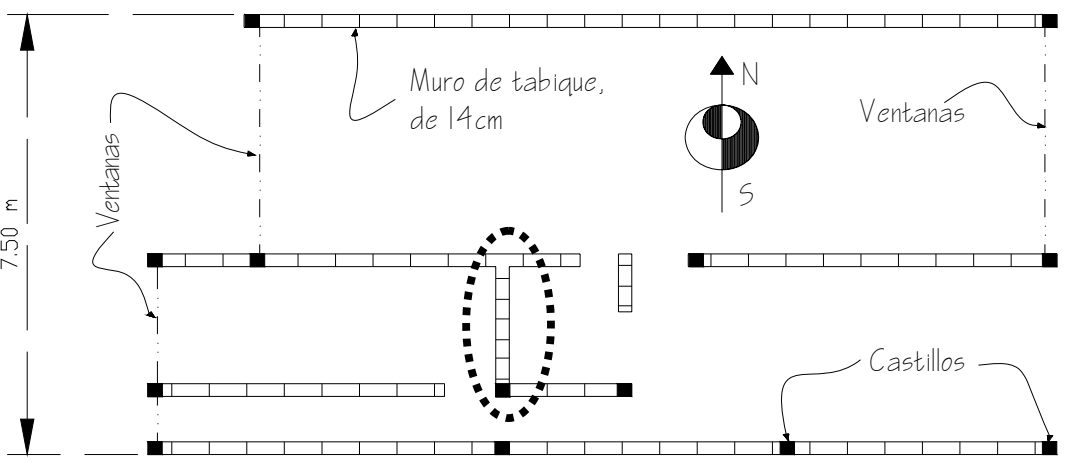

Figura 5. Plano de planta baja de la casa en la cerrada de Romero de Terreros \# 52. Solo tenía un muro de $2.5 \mathrm{~m}$ en el sentido Norte-Sur (Marsal, 1958).

\section{Colapsos parciales}

A continuación se describen brevemente los casos que sufrieron colapso parcial de las estructuras mostradas de la tabla 1:

- Edificio en Insurgentes \# 337 esquina con Coahuila (57-Cant, fig. 3 y tabla 1). Estructura de marcos de concreto reforzado de 5 pisos, propiedad del actor mexicano Mario Moreno "Cantinflas", solo quedó en pie la planta baja (fig.6a).

- Cine Encanto (57-Enca, fig. 3 y tabla 1). Ubicado en la calle de Serapio Rendón, estructura con columnas de concreto reforzado y armaduras de acero, el techo y parte de los muros se vinieron abajo (fig. 6b). Alrededor de 32 salas de diversión con sistemas estructurales similares fueron cerrados por seguridad. 
- Mercado de la Merced (57-Merce, fig. 3 y tabla 1). Estructura con cubierta formada por 94 cascarones de concreto reforzado, apoyados cada uno en pares de columnas de concreto reforzado empotradas en cajones, también de concreto reforzado con dimensiones de excavación que compensaban el peso propio de la estructura; seis de los cascarones de concreto se vinieron abajo (fig. 7).

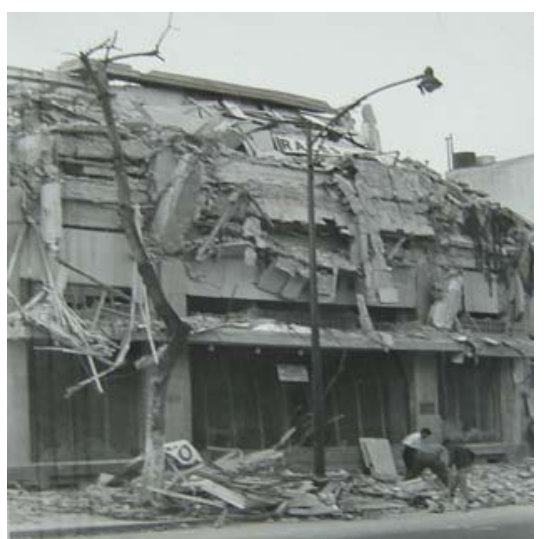

a)

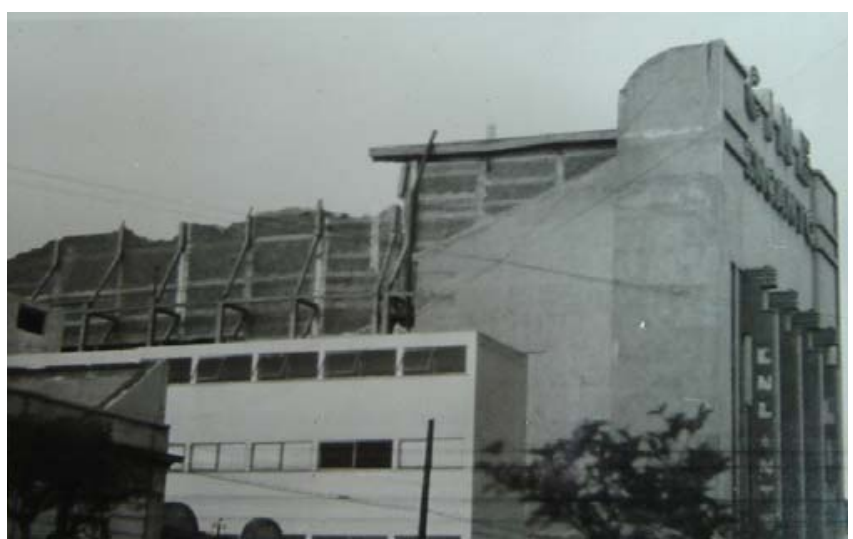

b)

Figura 6. Colapsos parciales: a) edificio en Insurgentes \# 377, sólo la planta baja quedó en pie y b) Cine Encanto que perdió el techo y parte de los muros.
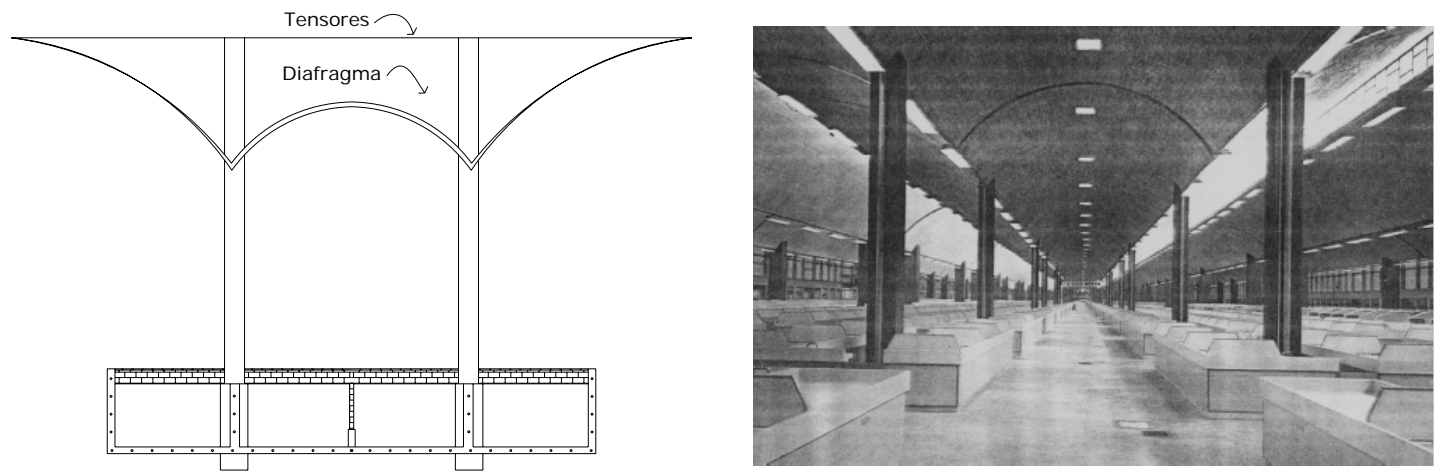

Figura 7. Mercado de la Merced, sufrió la caída de una parte de la cubierta (a la izquierda esquema de la estructuración original, Marsal, 1958)

- Edificio en calle Geranio esquina con Abedules en la colonia Atlampa (57-Gera, fig. 3 y tabla 1). Estructura de cinco pisos, sufrió el derrumbe de los niveles superiores; en él estaban las oficinas de "Elevadores Schindler".

- Inmuebles de "Muebles y Mudanzas" en Insurgentes \# 1338 y el vecino de embotelladora "Canadá Dry" (57-46, tabla 1). Estructuras formadas por dos cuerpos, al frente por oficinas de un piso con muros de concreto reforzado y en la parte posterior por bodegas de muros de tabique con castillos de concreto reforzado y armaduras de acero; el cuerpo de oficinas fue el que se derrumbó.

A pesar de los daños mencionados, el sismo no fue considerado una catástrofe pero toda la población quedó atemorizada, y en seguida se empezó a trabajar en la reconstrucción de la ciudad. En el ámbito de la Ingeniería se inició la búsqueda de respuestas del comportamiento de las estructuras y del 
suelo de la ciudad de México, así como la lucha contra los falsos profesionistas (fig. 8): "Los colegios de Ingenieros Civiles y Nacional de Arquitectos se solidarizaron ayer al pedir a las autoridades que acaben con la plaga de 'FIRMONES', habilitados como peritos constructores..." (Excelsior, 1957); éste, es un problema vigente.

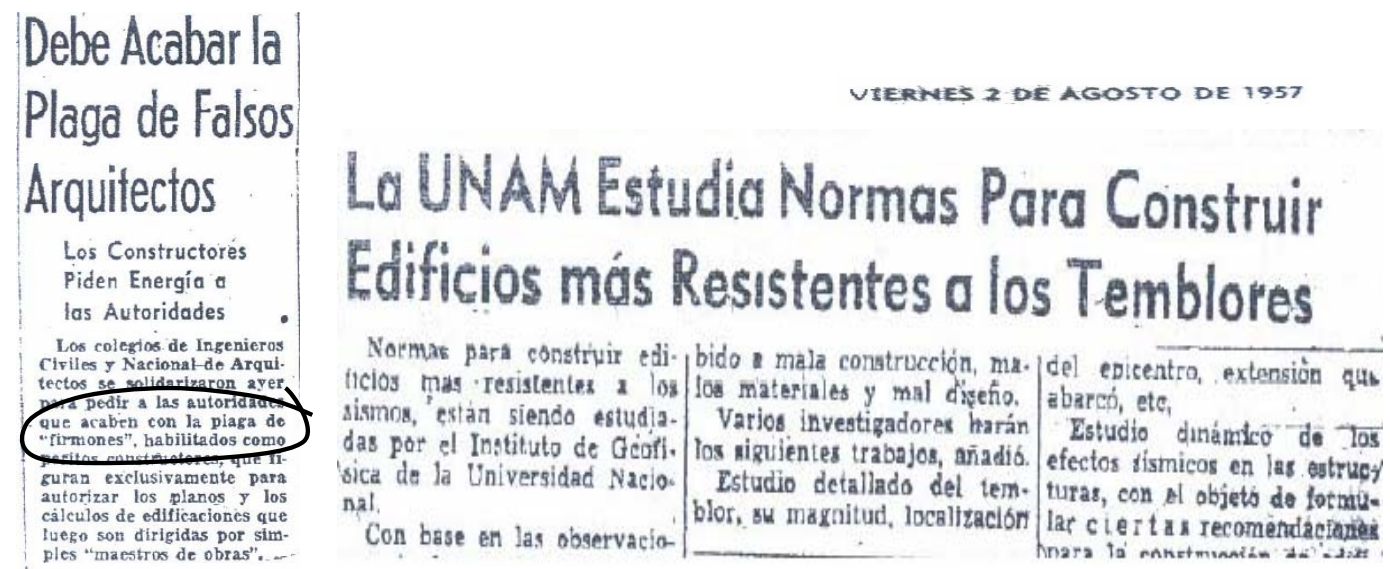

Figura 8. Fragmentos de reportajes publicados en Excelsior el 1 de Agosto de 1957, muestran las iniciativas que se tomaron en el área de la investigación y de la práctica; nótese en la izquierda el texto que critica la "plaga de firmones".

\section{ESTUDIO ESTADÍSTICO DE LOS DAÑOS}

Se estudiaron las estructuras de la tabla 1 con el propósito de conocer más sobre algunos de los factores que pudieron afectar su desempeño durante el sismo del 1957. En la fig. 9 se muestra la distribución de los daños durante el sismo: en el eje horizontal se tiene el nivel de daño y en los ejes verticales se evalúa el número de estructuras en cada caso y el porcentaje que representan del total. Como ya se mencionó, se aprecia que fueron pocos los colapsos y que los porcentajes en los niveles de daño leve, moderado y grave son muy similares. A continuación se presentan las distribuciones de daño en las estructuras considerando el tipo de sistema estructural, el golpeteo, la ubicación en esquina, irregularidad en planta y el número de niveles; más adelante se presenta un estudio que correlaciona el daño con el periodo dominante del suelo, los asentamientos diferenciales y el tipo de cimentación y algunos índices de intensidad sísmica.

\section{Número de niveles}

Las estructuras se clasificaron por número de niveles, para relacionarlo con el nivel daño, y se obtuvo la distribución mostrada en la fig. 10. En ella se observa que casi todos los colapsos y parte de los daños graves y moderados se presentaron en los edificios de baja altura, que en su mayoría tenían un sistema estructural de marcos de concreto, tipo nave industrial o mampostería confinada. En esa época el Reglamento de Construcciones del Distrito Federal no contemplaba el diseño sísmico de las estructuras con altura menor a $16 \mathrm{~m}$, que incluía estructuras hasta de cinco niveles, por lo que es muy probable que estas estructuras no hayan tenido un buen diseño estructural o una supervisión de ingenieros capacitados. Si observamos la categoría que contiene la suma de todas las magnitudes de daño (fig. 10), se aprecia que hay un valor máximo en las estructuras de cinco niveles: todas eran de marcos de concreto. En la misma categoría se observa que disminuyen los daños para las estructuras de seis niveles y van aumentando hasta llegar a las de once niveles, donde se tiene otro valor máximo de daños: nuevamente todas eran de marcos 
de concreto. Para las estructuras de doce niveles no se reportaron daños y para las de trece a dieciséis niveles hay menos daños y se observa que disminuyen conforme se aumenta el número de niveles, el sistema estructural varía, hay dos casos de construcción compuesta con daño leve y moderado, uno de marcos de acero con daño leve y el resto de marcos de concreto con daños moderados y graves. En esa época ya existía la Torre Latinoamericana construida a base de marcos de acero, tenía 43 niveles y el proyecto fue realizado con la participación de los mejores de la ingeniería mexicana de esa época; no fue reportada con daños.

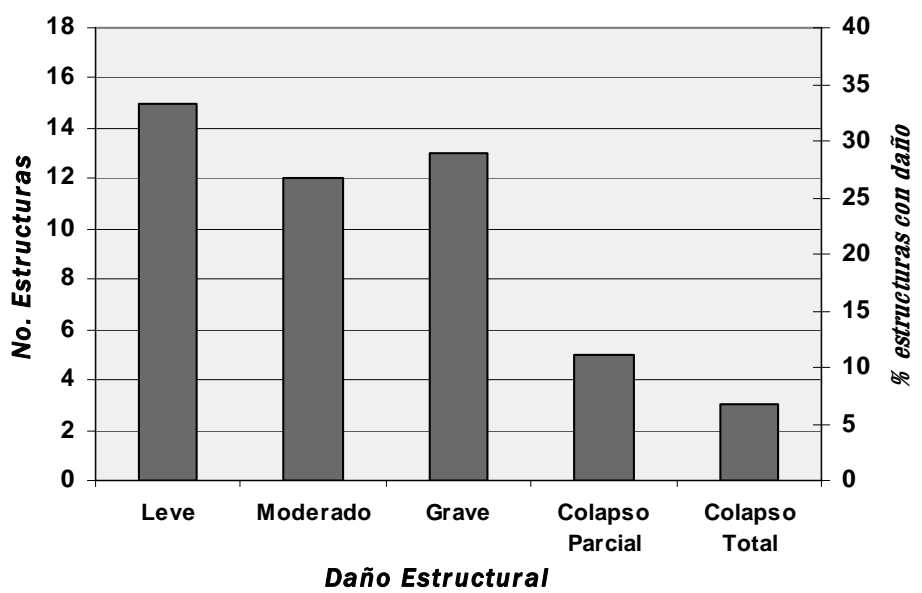

Figura 9. Comportamiento de estructuras de la tabla 1 durante el sismo del 28/07/57 considerando la magnitud del daño.

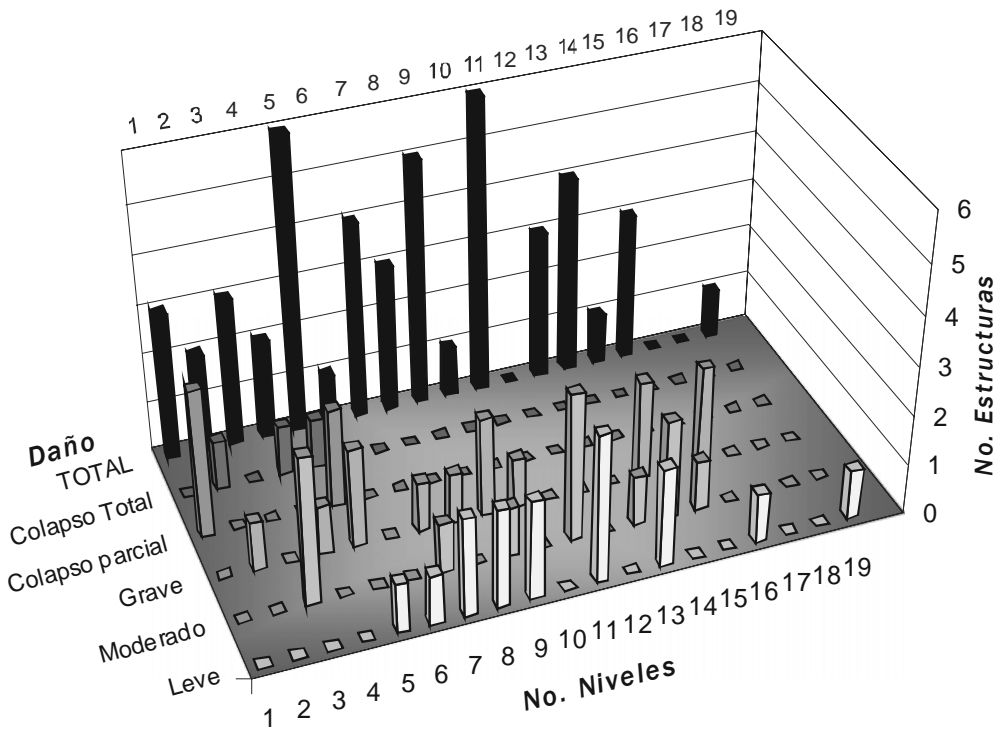

Figura 10. Comportamiento de las estructuras de la tabla 1 durante el sismo del 28/07/57 clasificadas por número de niveles y magnitud del daño. 


\section{Tipo de sistema estructural}

Tomando en cuenta la clasificación de las estructuras según el tipo de sistema estructural (tabla 1), se obtuvo la distribución de daños mostrada en la fig. 11. Se observa que las estructuras de marcos de concreto representan la mayoría de los daños de la muestra; se tienen dos colapsos totales y un parcial en este grupo. En las estructuras de marcos de acero se aprecia solo un caso con daño grave, no se tienen reportes de niveles de daño menores, ya que probablemente no presentaron daños importantes como se menciona en Excelsior (1957) "...las estructuras de acero han pasado victoriosamente la prueba del temblor del día 28..." (fig. 12). En las estructuras de construcción compuesta se observa solo un caso de daño moderado y uno de daño leve, esto muestra que aparentemente tuvieron buen comportamiento y que el concreto que recubría los marcos de acero aportó una resistencia extra, ya que no fue diseñado específicamente para esta función. En la mampostería confinada se aprecian tres casos de daño moderado, y el colapso total que se observa es el caso ya descrito de la casa que carecía de muros en la dirección N-S y la falla fue fuera del plano (57-S/M, tabla 1), es decir, como la falla típica de un muro aislado. En el sistema de naves industriales se observan dos colapsos parciales y un daño grave, la mayoría de los daños fue producto de la caída de la cubierta y de la falla de muros al no tener confinamiento suficiente; este sistema se utilizaba mucho en las salas de diversión (cines, casinos, salones de baile) y en bodegas. Muchas fueron cerradas para su revisión, pero no se tienen reportes de los dictámenes. En estos dos últimos tipos de sistemas estructurales no se tienen casos de daño leve o moderado, es muy probable que sí hayan ocurrido daños solo que no se encuentran reportados.

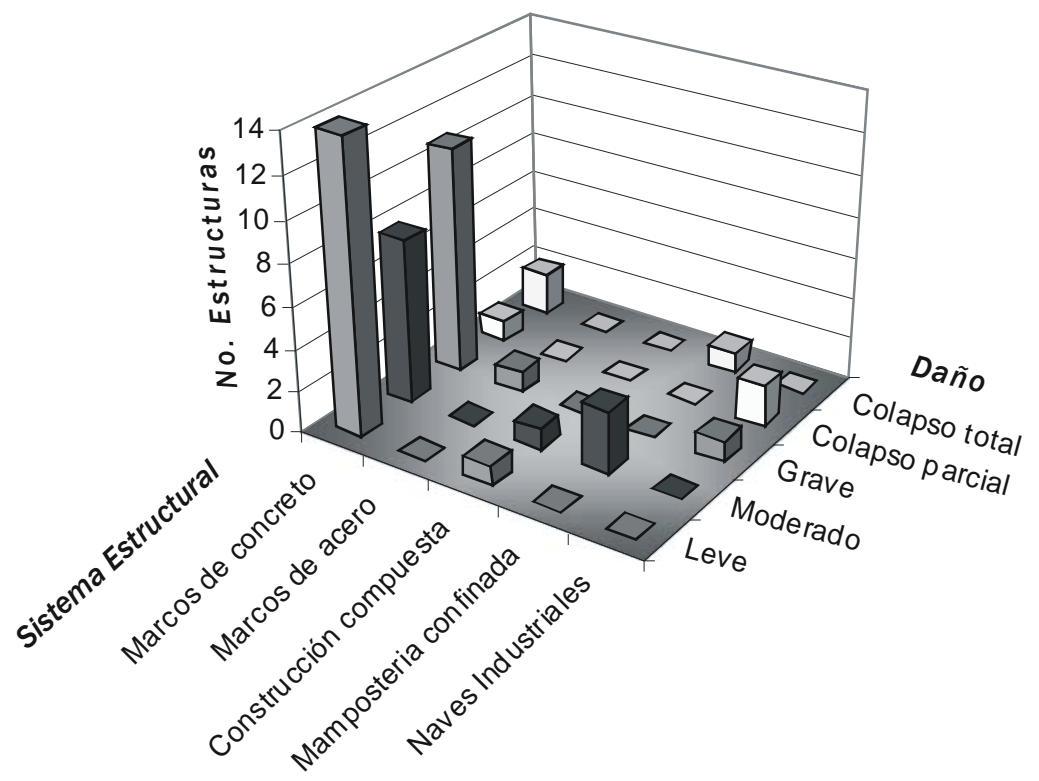

Figura 11. Comportamiento de las estructuras de la tabla 1 durante el sismo del 28/07/57 clasificadas por sistema estructural y magnitud de daño.

Para tener un comparativo entre los sistemas de estructuración que se utilizaban en el centro de la ciudad de México y la distribución de daño mostrada en la fig. 11, se realizó una encuesta a varios ingenieros expertos que conocen las construcciones de esa época. Los resultados nos indicaron que se construía con marcos de acero aproximadamente un 30\% de los edificios de seis a diez niveles, un $40 \%$ de los de once a quince niveles y un $60 \%$ de los de dieciséis o más niveles; esto aclara que sí existían varios edificios de acero y que este sistema estructural tuvo un buen desempeño, ya que se reportaron muy pocos casos de daño (incluyendo los de construcción compuesta). Cincuenta por ciento de los edificios de tres a 
cinco niveles se construían con marcos de concreto, el hecho de que todos los edificios entre cinco y once niveles reportados con daño eran de este tipo de sistema estructural, hace sobresalir un mal comportamiento no solo para este número de niveles sino para todos en general al tener una gran cantidad de casos reportados de este sistema (fig. 11). Para los edificios de uno y dos niveles resultó que predominaba la construcción con dos tipos de sistemas: mampostería confinada (43\%) y mampostería sin confinar (38\%), a pesar de ser elevado el porcentaje, se tuvieron pocos casos reportados de estos sistemas (fig. 11); es muy probable que por ser estructuras de bajo periodo estructural no hayan sido dañadas significativamente por el sismo y por lo tanto este sistema tuvo un comportamiento aceptable. El porcentaje restante de edificios de uno y dos niveles (20\%) se construía con marcos de concreto, tipo nave industrial y adobe; por lo tanto, existían pocas edificaciones con sistema estructural de nave industrial, sin embargo y como ya se comentó, en la fig. 11 se observan varios casos con magnitudes altas de daño de este sistema, lo que nos muestra que éste tuvo mal comportamiento.
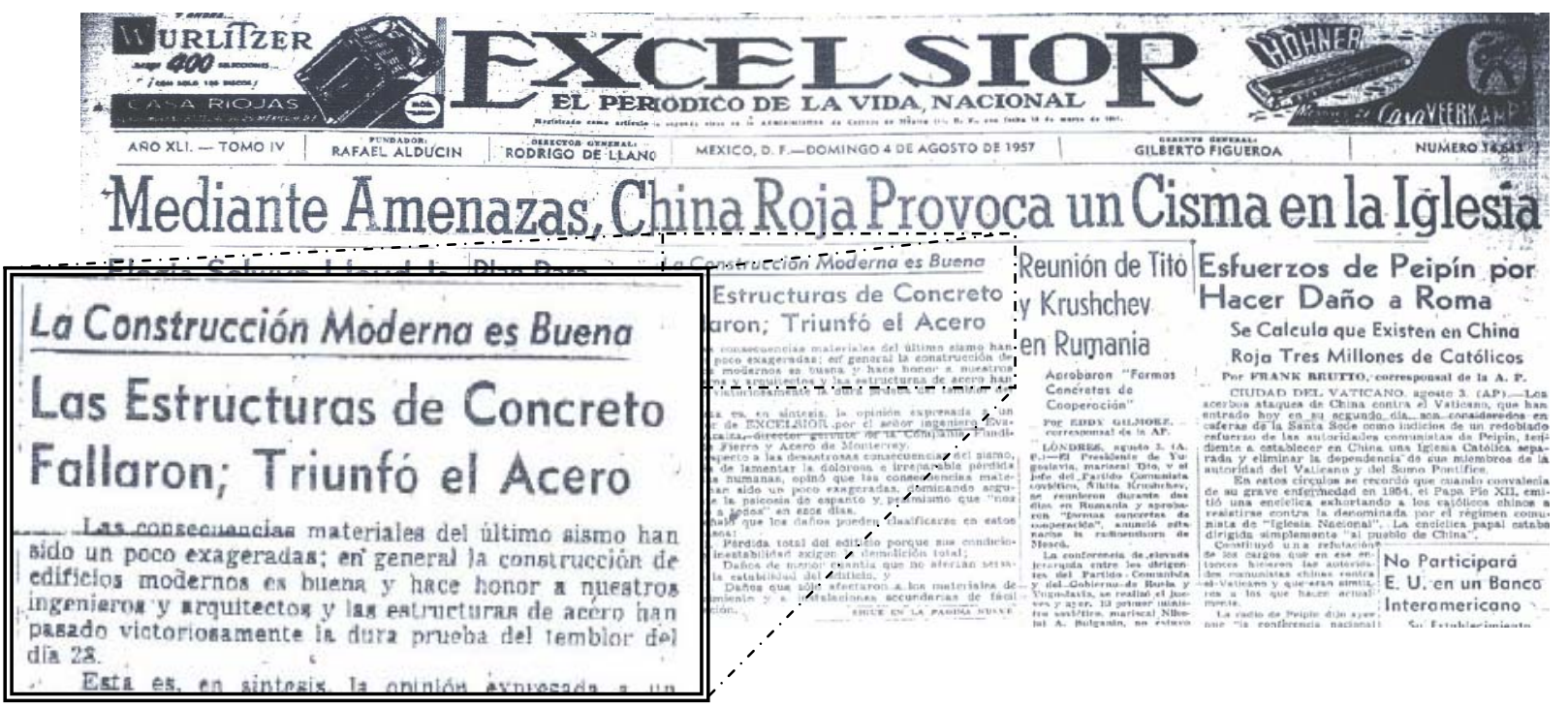

Figura 12. Publicación del Excelsior (1957) expresando que las construcciones de acero fueron más resistentes durante el temblor del 28/07/57, según la opinión del Ingeniero Evaristo Araiza, gerente de la Compañía Fundidora de Fierro y Acero de Monterrey.

\section{Golpeteo}

El efecto de golpeteo se considera que se presenta cuando la separación entre dos estructuras de diferente altura o rigidez no es lo suficientemente grande como para evitar el impacto entre ambas, al ocurrir desplazamientos producidos durante un sismo fuerte. Con respecto a este efecto de golpeteo, se tienen sólo cuatro casos claros que sufrieron impacto con edificios vecinos: 57-15, 57-53, 57-76 y 57-86 (tabla 1). En estas estructuras resalta que los daños importantes no se presentaron en todo el edificio sino solamente en las zonas de colindancias con las edificaciones vecinas, sin embargo, las descripciones de los daños no indican tener características de haber sido producto del impacto. La ocurrencia de pocos casos de golpeteo se puede atribuir a que en esa época había poca irregularidad en la altura de las construcciones, que había pocas estructuras de gran altura que pudieran presentar problemas de flexibilidad y que predominaban sistemas estructurales rígidos a base de muros. En la fig. 13 se muestra una vista panorámica del centro de la ciudad en 1957 donde se aprecian que existían pocas estructuras de gran altura. 


\section{Ubicación en esquina}

Se considera que una estructura está en esquina cuando los muros colindantes con las edificaciones vecinas son mucho más resistentes y rígidos que los marcos de las fachadas y no están adecuadamente desligados unos de los otros (fig. 14a). Durante un sismo fuerte la diferencia de rigidez entre fachadas y muros colindantes produce efectos de torsión y desplazamientos muy diferentes en los elementos de la estructura causando daños graves.

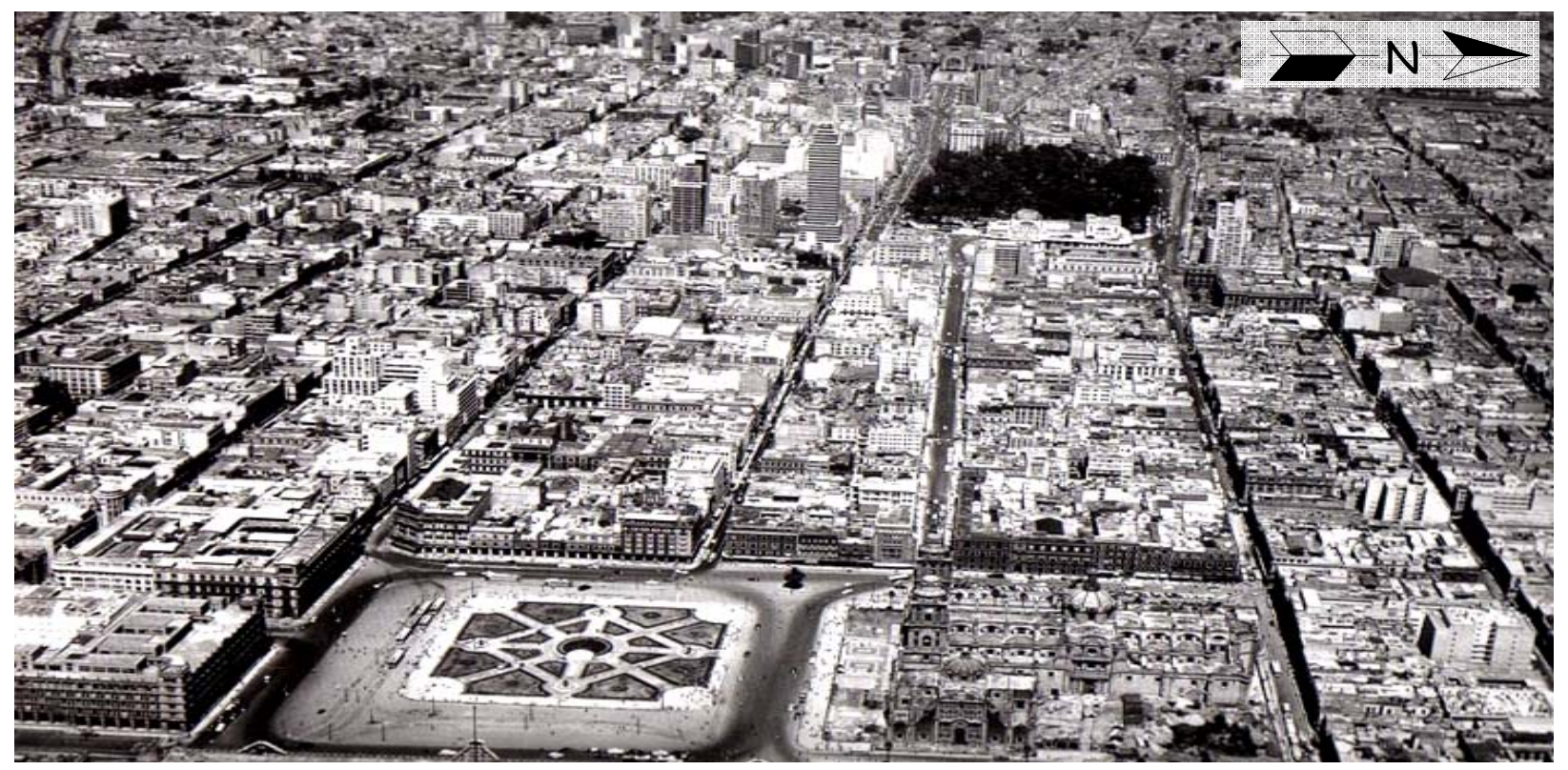

Figura 13. Vista panorámica del centro de la ciudad de México en 1957. Existían pocas estructuras de gran altura y había poca irregularidad en la altura de edificios.

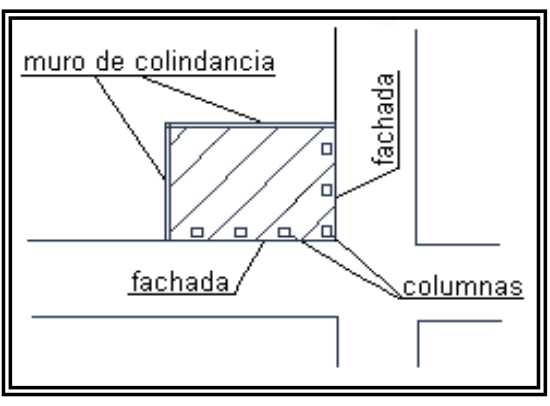

a)

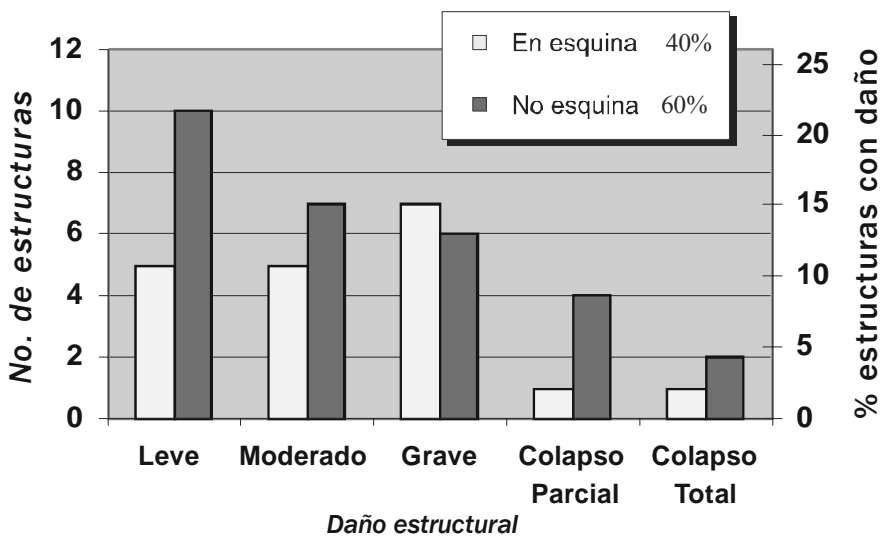

b)

Figura 14. a) Croquis típico de una de estructura de esquina y b) Comportamiento de las estructuras de la tabla 1 durante el sismo del 28/07/57 clasificadas por su ubicación en esquina y magnitud de daño. Se aprecia que el $40 \%$ estaba en esquina y la mayoría presentaron magnitudes altas de daño. 
Tomando la clasificación de las estructuras según lo anterior se obtuvo la distribución de daños mostrada en la fig. 14b donde se observa que dos estructuras de cada cinco (40\%) de la tabla 1 estaban en esquina, y los niveles de daños que tuvieron fueron altos, incluyendo un colapso total.

\section{Irregularidad en planta}

Se considera irregularidad en planta cuando existe, ya sea desde el punto de vista estructural o arquitectónico, distribución asimétrica de elementos resistentes y de la masa de la estructura provocando que el centro de masa y el centro de torsión no coincidan; esto durante un sismo induce efectos de torsión causando mayores desplazamientos y esfuerzos y por lo tanto daños graves en las estructuras. Tomando en cuenta lo anterior se clasificó la muestra y se obtuvo la distribución de daños mostrada en la fig. 15a. En ella se observa que la mitad de las estructuras reportadas con daño tenían irregularidad en planta. Se aprecia que muchas de estas estructuras tuvieron daño leve, sin embargo, también se presentaron dos casos de colapso parcial y uno de colapso total. Además de lo mencionado en el tema anterior, las estructuras en esquina son casos muy frecuentes donde se presenta también irregularidad en planta. En la fig.15b se muestra el edificio Corcuera (57-Corcu, tabla 1 y fig. 3), conocido por su anuncio de Goodrich Euzkadi y su enorme llanta en lo alto. Este edificio era una estructura de esquina, que aunque no se aprecia en la imagen, tenía en la parte posterior un muro de rigidez; además, la esquina era en diagonal lo que agravó la irregularidad en planta; los daños que presentó este edifico durante el sismo fueron tan severos que causaron su demolición meses después. En la fig. 15c se muestra en la misma esquina la estructura actual; a pesar de ser más moderna es muy similar en la irregularidad, tanto en planta como en altura.

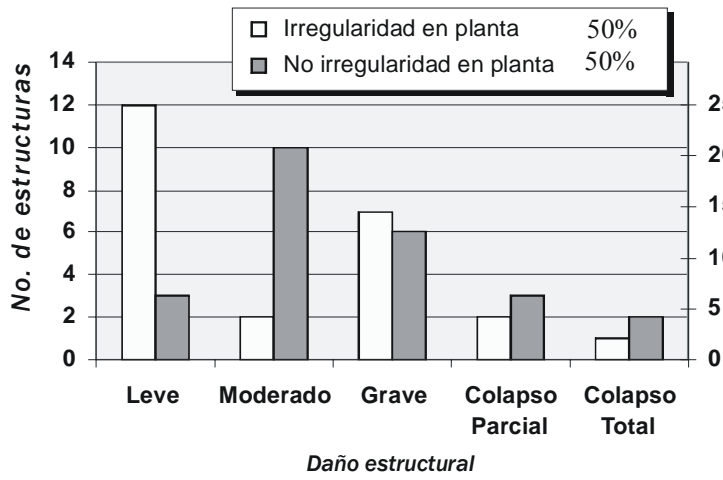

a)

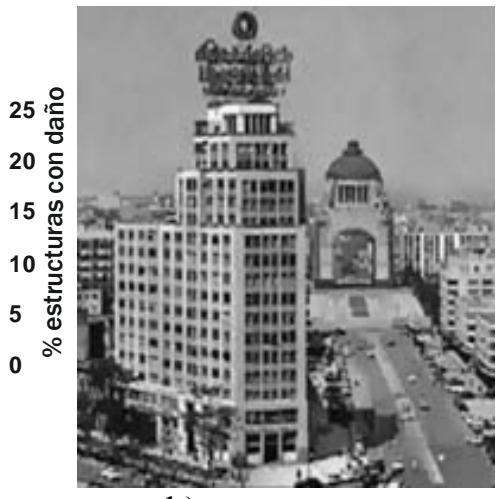

b)

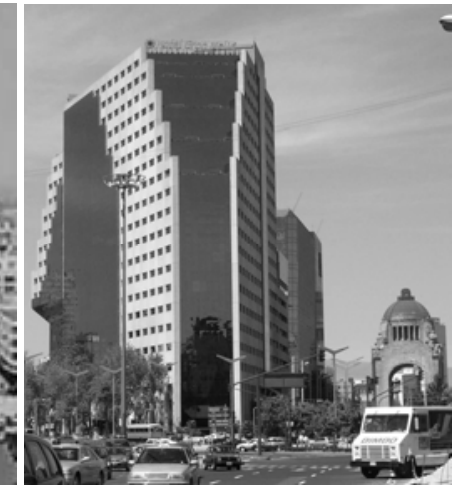

c)

Figura 15. a) Comportamiento de las estructuras de la tabla 1 clasificadas según su irregularidad en planta y daño estructural, 50\% de las estructuras tenían planta irregular, en b) edificio Corcuera (57-Corcu en tabla 1) en esquina y con planta muy irregular por encontrarse en un cruce de calles en diagonal, fue demolido después del sismo por los daños tan severos que sufrió y en c) estructura actual en la misma esquina muy similar a la anterior.

De las estructuras en esquina estudiadas sobresale, como en el caso del edificio Corcuera, que aproximadamente la mitad tenían mucha irregularidad en planta debido a que se encontraban en cruces de calles en diagonal. Con ayuda de un SIG se localizaron este tipo de estructuras. En la fig. 16 se muestra parte del resultado: se aprecia un tramo de la Avenida Paseo de la Reforma, que por su orientación presentó varios casos como estos. Se muestran en el plano con color negro las estructuras 57-70, 57-74 y $57-C o r c u$ (tabla 1, fig. 3 y fig. 16) y se muestra en un croquis la forma en planta de las estructuras $57-70$ y 57-74, observándose la gran irregularidad en planta que tenían. Una foto panorámica de esa zona tomada antes del sismo muestra las estructuras mencionadas. 
Clasificando las estructuras que estaban en esquina por su irregularidad en planta se obtuvo la distribución de daños mostrada en la fig. 17. Se observa que un poco más de la mitad tenían mucha irregularidad en planta y que los daños en su mayoría fueron de intensidad grave. Es muy probable que los efectos de torsión se hayan incrementado y provocaron daños más intensos en estas estructuras que en las que no tenían tanta irregularidad. Para los casos de estructuras de esquina con colapso parcial o total no se tiene certeza de la poca o mucha irregularidad en planta, por lo que no se consideraron en la obtención de la fig. 17.

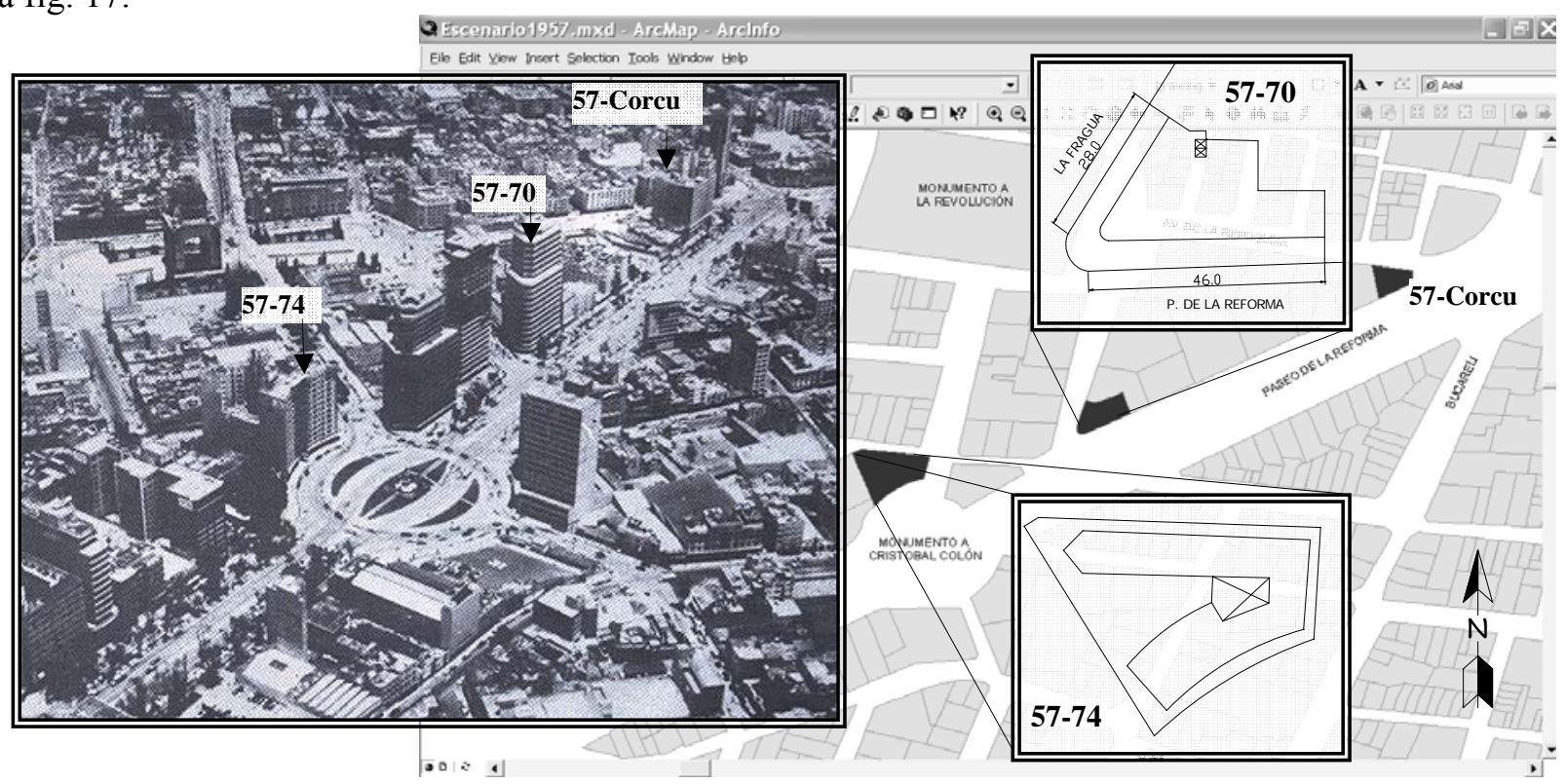

Figura 16. En la Av. Paseo de la Reforma debido a su orientación había varias estructuras en cuchillas, con bastante irregularidad en planta. Se muestran esquemáticamente con ayuda de un SIG y en fotografía tres casos de estructuras en esquina y con planta muy irregular.

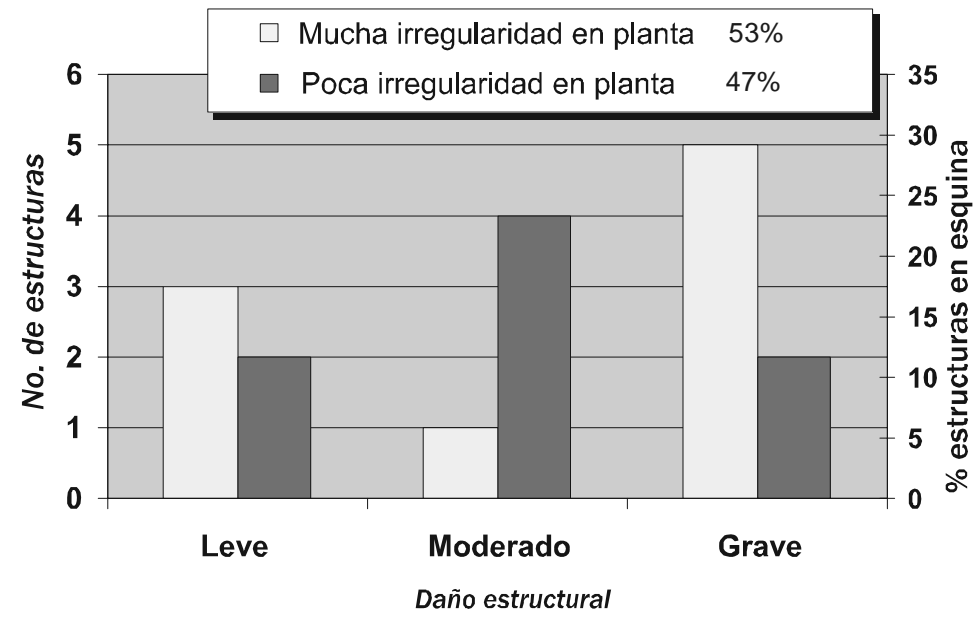

Figura 17. Comportamiento de las estructuras en esquina de la tabla 1 durante el sismo del 28/07/57, clasificadas por su irregularidad en planta y magnitud de daño. Nótese que la mayoría de las estructuras que tuvo daño grave tenía mucha irregularidad en planta. No se tomaron en cuenta los casos de estructuras con colapsos debido a que no se conoce esa información. 


\section{Asentamientos diferenciales y tipo de cimentación}

Para asociar el efecto del tipo de cimentación de las estructuras con la magnitud del daño estructural primero se clasificó la muestra tomando en cuenta el daño que sufrió dicha cimentación; en este caso por la información disponible solo se conoce si sufrieron asentamientos diferenciales después del sismo, considerando éstos como hundimientos no uniformes en la base de la estructura que provocan desplome de la misma. Si la inclinación es leve puede causar pocos daños y solamente en elementos no estructurales, pero si es muy grande puede causar daños severos en elementos estructurales y además poner en peligro la estabilidad de la estructura. Se clasificó la muestra en cinco grupos (tabla 1): a) Pilotes de Madera (PM), b) Pilotes de Concreto (PC), c) Cajón de Cimentación (CC), d) Parcialmente Compensada con cajón de cimentación y e) con Pilotes (PCP) y Superficial (S); no se consideraron seis estructuras de la tabla 1 al no tener los datos de su cimentación y desafortunadamente están entre ellos un caso de colapso total y tres parciales. En la fig. 18a se muestra la distribución obtenida considerando lo anterior, se observa que las estructuras con pilotes de madera y cimentación superficial son las que presentaron más asentamientos después del sismo, aunque a su vez son las más numerosas.

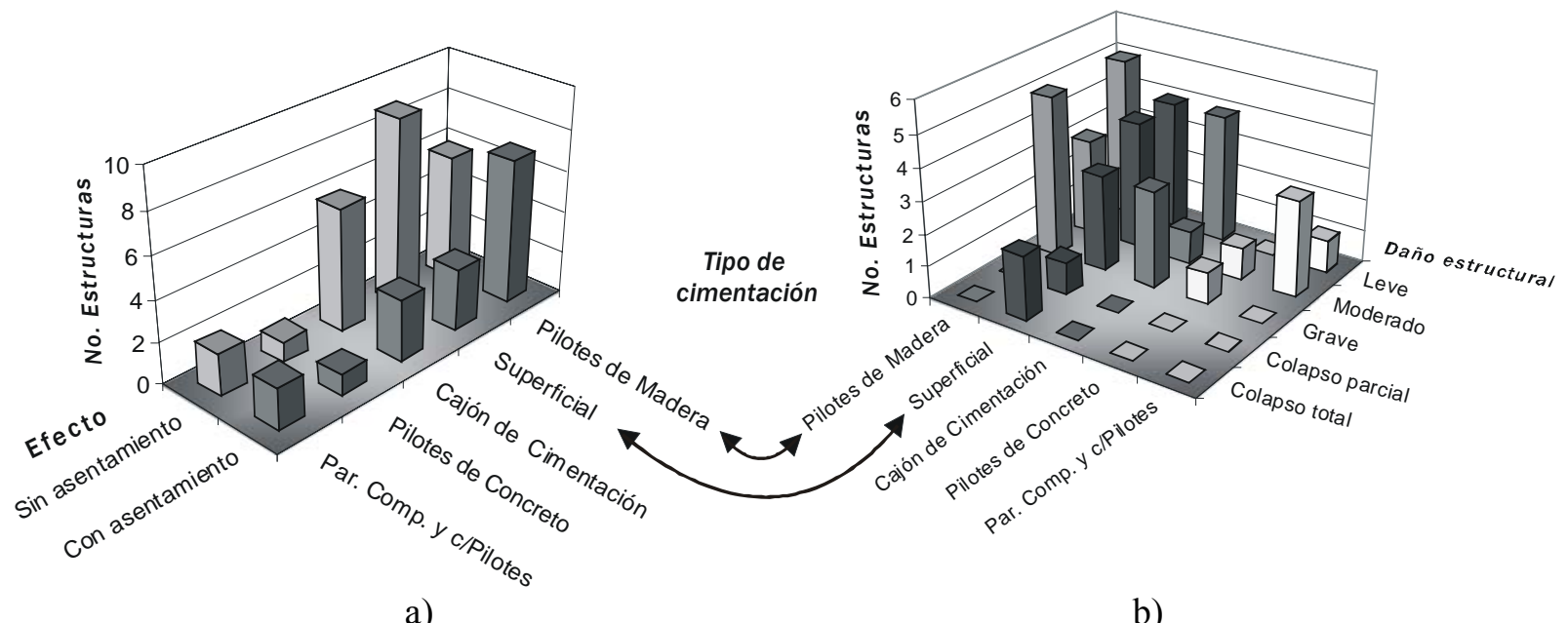

Figura 18. Comportamiento de estructuras de la tabla 1 durante el sismo de 28/07/57 clasificadas en a) por el tipo de cimentación y por presencia de asentamientos diferenciales despúes del sismo y en b) por el tipo de cimentación y la magnitud de daño estructural.

En la fig. 18b se muestra la distribución al considerar el daño estructural y el tipo de cimentación. Se aprecia que las estructuras con cimentación superficial, pilotes de madera y cajón de cimentación representan la mayoría de la muestra, sin embargo, los casos de colapso parcial y total en que sí se tenía información completa se presentaron únicamente en la categoría de cimentación superficial, que junto con la cimentación de pilotes de madera son las que tuvieron la mayoría de los daños de magnitud moderada y grave. El cajón de cimentación presentó en su mayoría casos de daño leve y muy pocos de daño moderado y grave. De los tipos de cimentación con pilotes de concreto y parcialmente compensada se tienen pocos casos registrados de daño y éstos son de magnitud leve, moderada y grave. En las figs. 18a y 18b se aprecia que la cimentación de pilotes de madera, junto con la superficial, presentaron la mayoría de los casos con asentamientos diferenciales y también la mayoría de los casos con magnitud de daño grave a colapso total y, aunque ya se mencionó que eran los tipos que más habían, sugiere una correlación entre asentamientos diferenciales y daño estructural.

En la fig. 19a se muestra el comportamiento de las estructuras de la tabla 1 clasificadas por la ocurrencia de asentamientos diferenciales y daño estructural. Se observa que dos estructuras de cada cinco 
(40\%) presentaron asentamientos y además este porcentaje se mantiene uniforme tomando en cuenta las distintas magnitudes de daño (se omitió la categoría de colapso total ya que en este caso no se pudo determinar si hubo asentamientos en la estructura). En la fig. 19b se muestra la distribución obtenida al clasificar sólo a las estructuras con cimentación de pilotes de madera, siguiendo el mismo criterio de la fig. 19a. Se observa que un poco más de la mitad (54\%) de las estructuras con este sistema tuvieron asentamientos y éstas presentaron en su mayoría daños intensos. Se hizo lo mismo para los otros sistemas de cimentación y se obtuvieron los porcentajes de casos con asentamientos diferenciales siguientes: cimentación superficial $29 \%$, cajón de cimentación 33\%, parcialmente compensada con cajón y pilotes $50 \%$ y pilotes de concreto $50 \%$. Se observa que los porcentajes de presencia de asentamientos son significativos (sin olvidar que es pequeño el conjunto observado), sobresaliendo las cimentaciones más antiguas como los pilotes de madera.

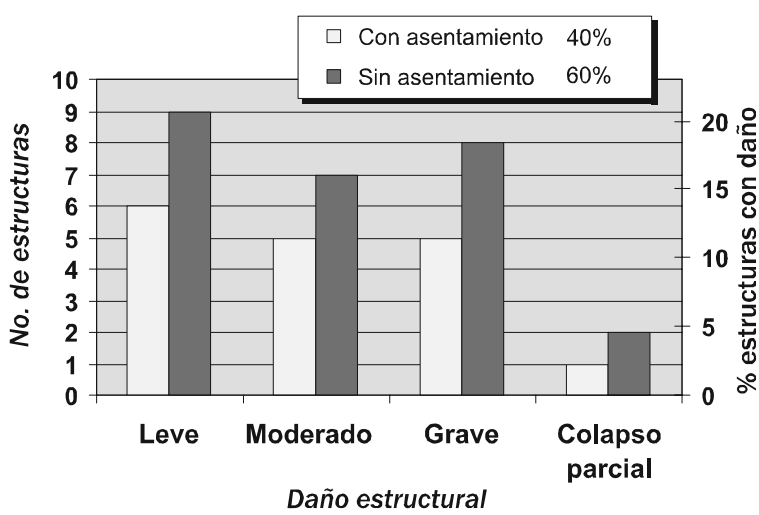

a)

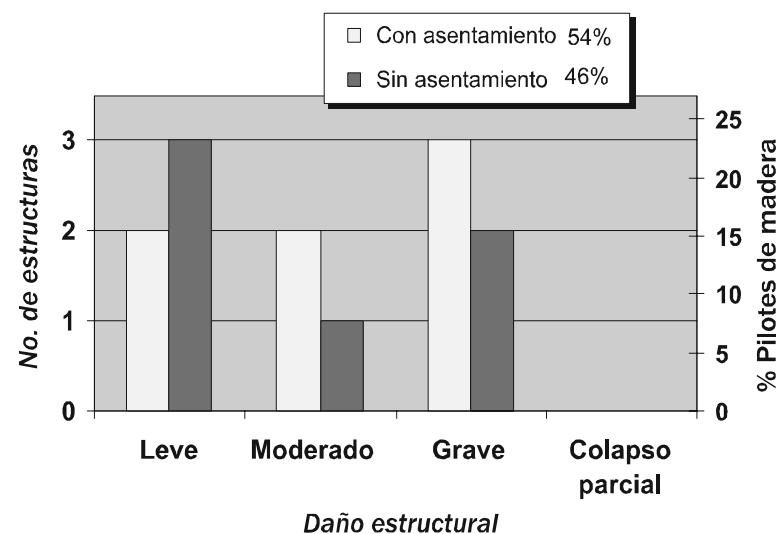

b)

Figura 19. Comportamiento de estructuras en a) de la tabla 1 durante el sismo de 28/07/57 clasificadas por la presencia de asentamientos diferenciales y magnitud de daño estructural despúes del sismo y en b) solo

las de cimentación con pilotes de madera clasificadas con el mismo criterio. Nótese el porcentaje significativo de estructuras que presentaron asentamientos diferenciales.

\section{CORRELACIÓN ENTRE LA MAGNITUD DEL DAÑO ESTRUCTURAL Y LAS PROPIEDADES DEL SUELO}

El suelo es uno de los principales factores que intervienen en el comportamiento sísmico de las estructuras. En este trabajo se pretende analizar algunos de sus efectos que pudieron influir en el daño de las construcciones.

\section{Intensidad Sísmica}

Si se conoce el nivel de intensidad del movimiento del suelo se puede determinar la demanda sísmica para un evento. Esta intensidad se acostumbra describir por medio de tres parámetros: la aceleración máxima, la velocidad máxima y el desplazamiento máximo de la estructura, debido a que la respuesta estructural es sensible a cada uno de éstos dependiendo de sus propiedades dinámicas. El camino más sencillo es hacerlo a través del cálculo de la respuesta de un oscilador de un grado de libertad e ir formando espectros de respuesta. En este trabajo para obtener los índices mencionados se utilizó el programa de computo "Z" (Ordaz y col., 1997); se calculó en coordenadas geográficas una malla de la Delegación Cuauhtémoc en donde cada punto contiene datos en términos de espectros de aceleraciones. Estos datos son resultado de utilizar los registros de un sismo histórico (24/10/93 Copala, Gro.) con 
epicentro muy cercano a la zona donde se originó el del 28/07/57 ya que no se cuenta con registros de este sismo. El programa permite escalar la magnitud del sismo escogido en este caso de $\mathrm{M}=6.7$ a $\mathrm{M}=7.5$ y la distancia epicentral de $\mathrm{R}=310 \mathrm{~km}$ a $\mathrm{R}=350 \mathrm{~km}$; con esto asumimos que este sismo escalado presenta intensidades muy similares a las que se pudieron presentar en 1957.

Se graficaron los espectros de respuesta, obtenidos como se explicó en el párrafo anterior, para los sitios que corresponden a la ubicación de las estructuras de la tabla 1. Se identificaron los periodos estructurales (Te) de cada una de ellas en estos espectros con el fin de observar la ordenada espectral para ese valor. El periodo estructural se obtuvo con la expresión de Mosquera y col. (2006). En la fig. 20a presenta el caso de la estructura 57-Corcu donde el periodo de ésta y el máximo espectral del sitio coinciden; otras dos estructuras de la tabla 1 estuvieron en este caso (57-74 y 57-69), todas éstas con daño grave. Las aceleraciones espectrales calculadas en estos casos van de 130 a 160 gals. Algunas estructuras de la tabla 1 tenían un Te mucho menor al del suelo (fig. 20b, estructura 57-Enca, y otras como 57-Merce y 57-55) donde se calcularon aceleraciones muy bajas, entre 30 y 40 gals; sin embargo, todas estas estructuras eran de tipo nave industrial con fallas estructurales graves asociadas a fallas de muros altos sin mínima resistencia lateral. Un caso intermedio a los dos anteriores (fig. 20c a la estructura 57-37) es cuando el Te es menor que el de las ordenadas máximas, lo que sugiere que la estructura al dañarse pierde rigidez modificando su Te, acercándolo a las ordenadas máximas. De lo anterior, sin tomar en cuenta los casos de tipo nave industrial, se concluye que como era de esperarse existe alguna correlación entre el daño y las ordenadas máximas en el espectro de respuesta. Sin embargo, cuando se incluyen todas las estructuras de la tabla 1 en el análisis (Orozco, 2006), se observa que en muchos casos de daño grave y colapso el Te de la estructura está muy por debajo de las ordenadas máximas con aceleraciones espectrales muy bajas y, por otro lado, estructuras sin daño o con daño leve fueron sometidas a grandes aceleraciones espectrales sin que esto se reflejara en su comportamiento.

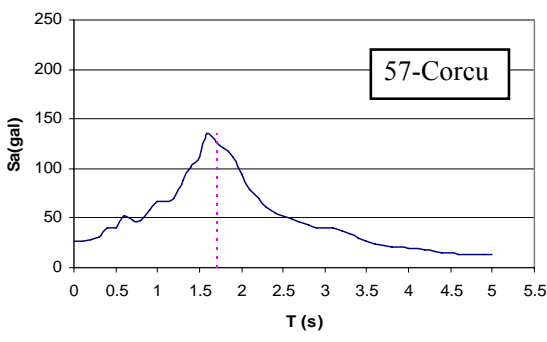

a)

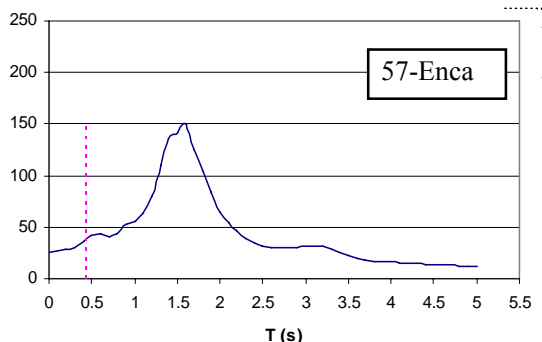

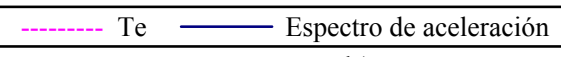

b)

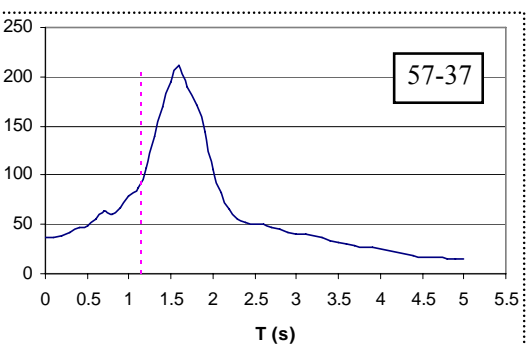

c)

Figura 20. Comparación del espectro de aceleración del sitio y el Te de las estructuras con magnitudes altas de daño: a) estructura 57-Corcu, el Te coincide con ordenadas máximas, b) estructura 57-Enca el Te está muy lejano a las ordenadas máximas, pero tenía sistema estructural tipo nave industrial que mostró mal comportamiento y c) estructura 57-37 el Te está antes de las ordenadas máximas.

Se obtuvieron varios mapas de intensidad de aceleración con ayuda de un SIG y los datos obtenidos del programa "Z", estos mapas son útiles para visualizar en qué zonas se presentaron los valores mayores de aceleración. En la fig. 21 se muestran mapas de intensidad de aceleración y la ubicación de las estructuras de la tabla 1; en la fig. 21a se muestra el mapa de aceleraciones máximas de cada sitio, aunque sabemos que éstas no se pueden presentar en el mismo instante, nos sirve para darnos una idea de las zonas que tuvieron valores mayores de aceleración. En la fig. $21 \mathrm{~b}$ se indica el mapa de aceleraciones correspondientes a $\mathrm{Te}=0.8 \mathrm{~s}$, en ambos mapas las zonas de aceleraciones mayores se muestran en tonos oscuros. Se aprecia que hay pocos casos en donde coinciden los daños con las aceleraciones máximas. Se realizaron mapas para otros períodos (Orozco, 2006) pero tampoco se encontró correlación. 
Utilizando los datos de velocidad máxima que también proporciona el programa “ $Z$ ” y con apoyo del SIG se realizó un mapa de intensidad de velocidad (Orozco 2006), pero tampoco se observó una coincidencia notable de los daños con las zonas de velocidades mayores. Para los desplazamientos también se realizaron mapas de intensidad para diferentes periodos, los valores se obtuvieron a partir del espectro de aceleraciones $\left(A=\omega^{2} d\right)$. En la fig. 22a se muestran los mapas de desplazamiento para los valores máximos espectrales de cada sitio y en la fig. $22 \mathrm{~b}$ para los valores con un Te de $1.5 \mathrm{~s}$, no se observa coincidencia de los daños con las zonas de valores máximos en ninguno de los dos casos mostrados.

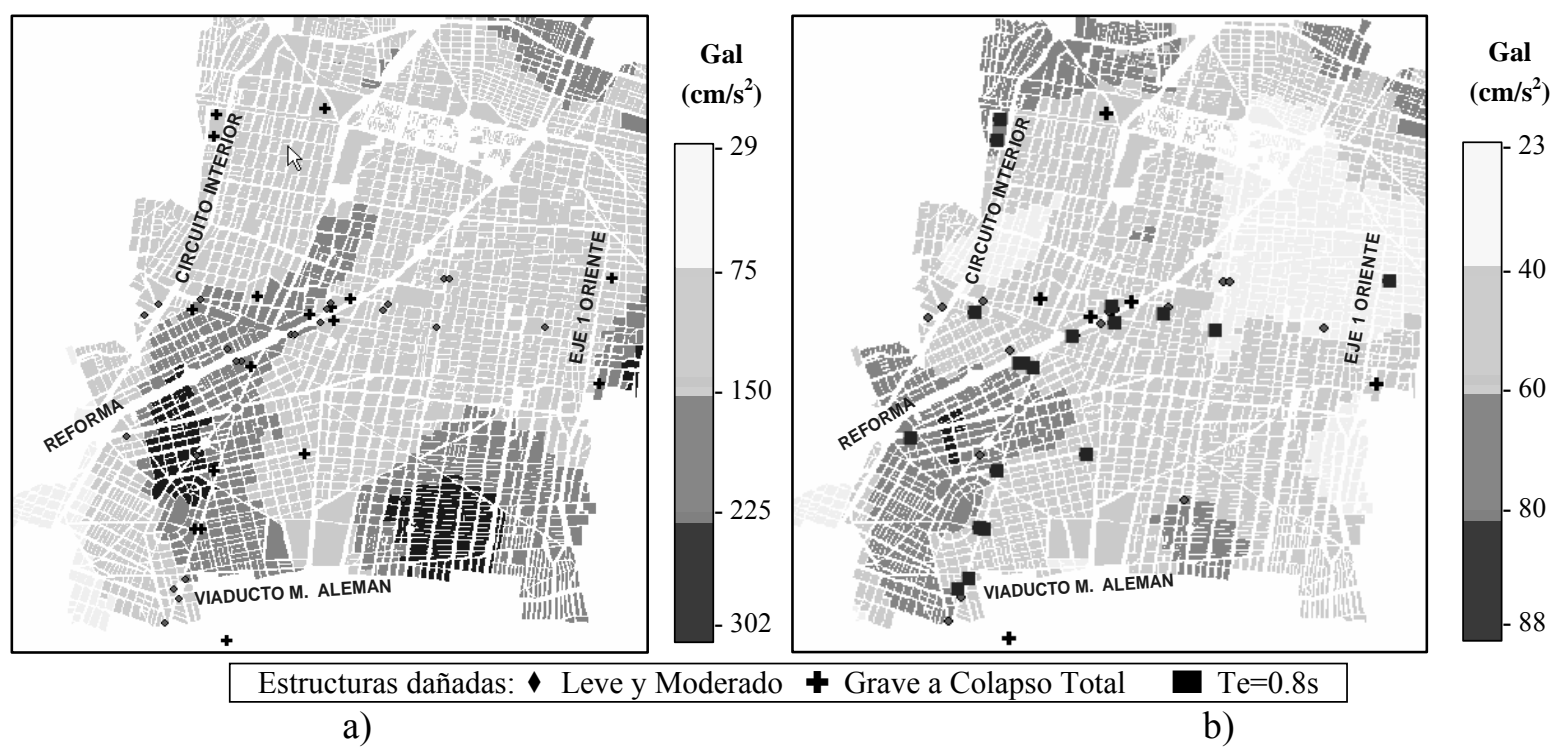

Figura 21. Estructuras con daño y mapas de aceleración a) para valores máximos espectrales de cada sitio y b) para valores de $\mathrm{Te}=0.8 \mathrm{~s}$. Se aprecian pocos casos en los que coinciden los daños con las zonas de mayores aceleraciones.

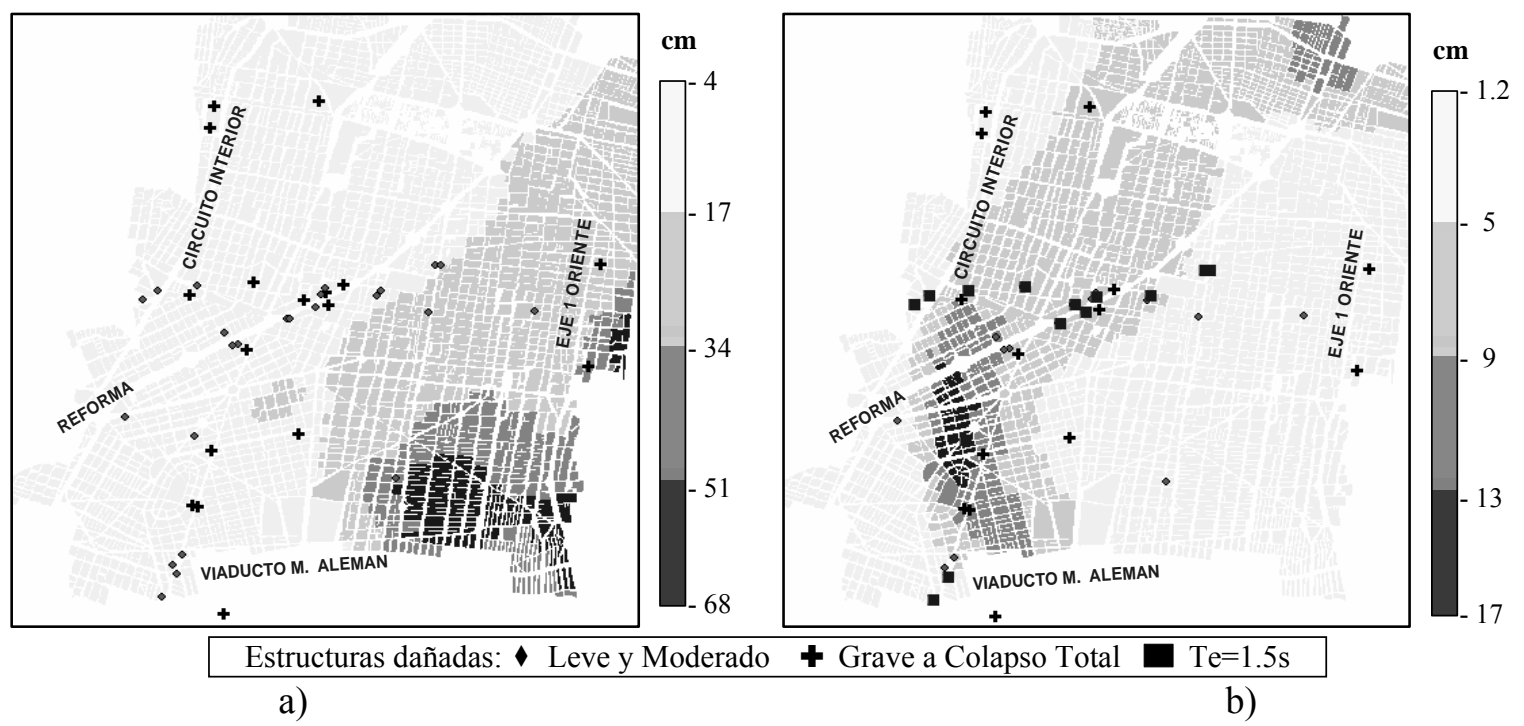

Figura 22. Mapas de intensidad de a) desplazamientos máximos espectrales para cada sitio y b) desplazamiento para $\mathrm{Te}=1.5 \mathrm{~s}$. Se aprecian pocos casos en los que coinciden los daños con las zonas de mayores desplazamientos. 
La distorsión de entrepiso que sufren las estructuras durante un sismo está fuertemente correlacionada con el daño estructural. Estas distorsiones se pueden estimar a partir de los desplazamientos obtenidos; se emplearon las expresiones de Miranda (1999) para la obtención de desplazamientos de azotea y distorsiones de entrepiso a partir del valor obtenido del espectro de desplazamientos y considerando que el primer modo de vibrar es el que domina. Debido a que las magnitudes de daño manejadas son sólo una apreciación y no una medida para poder obtener una correlación, sólo se realizó una comparación directa tanto para los valores de aceleración como para los de distorsiones. En la fig. 23a se muestra la comparación de la aceleración (eje horizontal) con el daño (eje vertical). En los valores de aceleración se tomó en cuenta que el cálculo del Te es incierto por lo que se eligió el valor máximo de aceleración espectral en un intervalo de $\mathrm{Te} \pm 0.5 \mathrm{~s}$. Nótese que se añadieron algunas estructuras con daño estructural nulo para observar de forma más completa la comparación. La tendencia esperada era que los puntos estuvieran por debajo de las líneas punteadas, de manera que las estructuras diseñadas con grandes resistencias pudieran mostrar poco daño ante grandes aceleraciones, y las menos resistentes estar justamente más cerca de dichas líneas. Las estructuras de la zona sombreada son las que fallaron ante aceleraciones espectrales muy bajas (menos de 80 gal) y es evidente que tuvieron errores constructivos inadmisibles por fallas de muros o de mampostería (55-55, 57-Merce, 57-Enca, 5790, 57-83). Hay que recordar que aunque estas estructuras no se pueden considerar fuera de reglamento, ya que lo que entonces se exigía como coeficiente de diseño eran 25 gal, es claro que prácticamente todas las estructuras resistieron más que eso, lo que es un ejemplo más de la generosa sobrerresistencia. En la fig. 23b se aprecia la comparación de las distorsiones de entrepiso con el daño, donde la tendencia es muy similar a la de Amax; obsérvese que las distorsiones calculadas son muy pequeñas, lo que habla de la baja calidad de las construcciones de la época.

\section{Periodo del suelo vs. Periodo estructural}

Se considera que el efecto de resonancia se presenta cuando en una excitación sísmica el periodo natural de una estructura es muy similar al periodo dominante del suelo sobre el que está desplantada. Después del sismo de 1957 se mencionó que la resonancia podría haber sido la causa de daño en varias de las estructuras (Excelsior, 1957); hipótesis que se ha mantenido desde entonces.

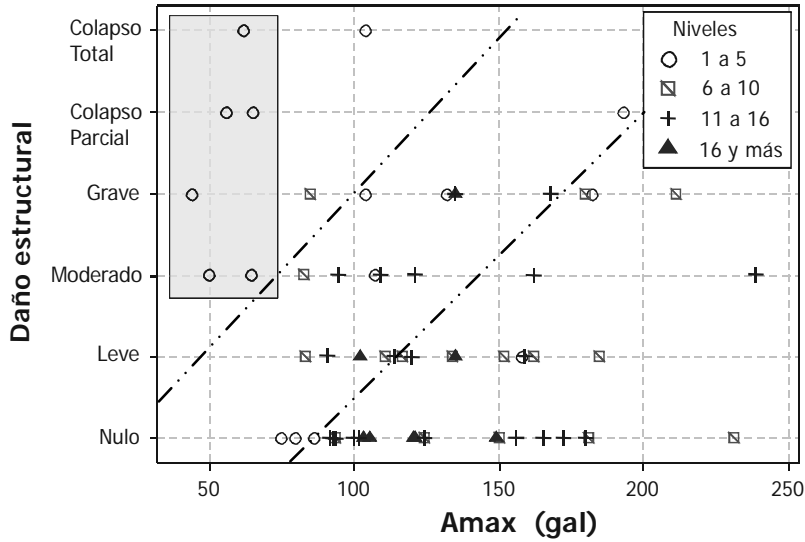

a)

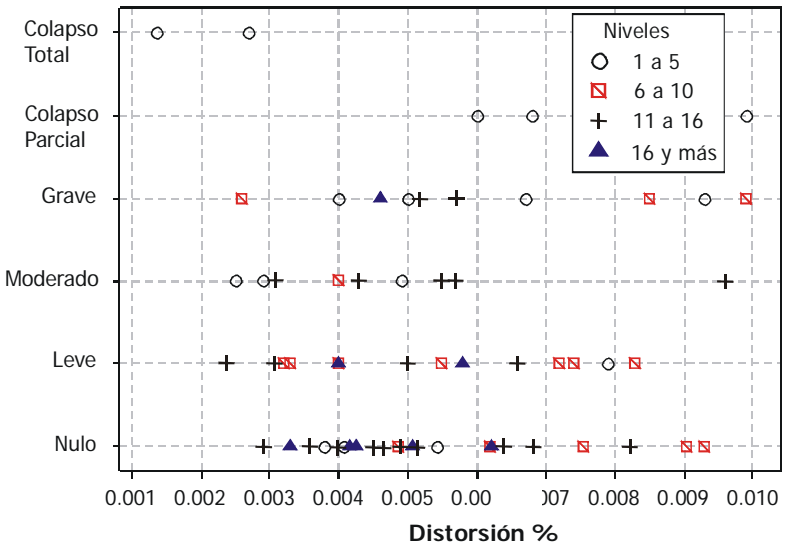

b)

Figura 23. Comparación de la magnitud de daño estructural a) con la aceleración espectral en $\mathrm{Te} \pm 0.5 \mathrm{~s}$ y

b) con las distorsiones de entrepiso evaluadas. No se aprecia ninguna tendencia, sólo se aprecia que no hubo daño debajo de los 80 gals omitiendo las estructuras de la zona sombreada. 
Con el fin de estudiar la posible resonancia se relaciona aquí el periodo del suelo con el número de niveles y con el periodo natural de las estructuras. Se obtuvo el periodo dominante del suelo para cada una de los sitios donde estaban desplantadas las estructuras con el programa de computo " $Z$ " (Ordaz y col., 1997). En la fig. 24 se muestra la correlación entre el número de niveles de las estructuras (eje horizontal) y el periodo del suelo (Ts, eje vertical), los puntos mostrados son con daño grave y colapso, y que debieron haber alcanzado una resistencia suficiente para llegar a la resonancia ya que no tuvieron problemas de golpeteo, fallas por defectos de construcción, fallas fuera del plano y algunos otros efectos que por las descripciones en los reportes resultaba claro que la resonancia no era necesariamente el motivo del daño. La correlación obtenida es pobre $\left(\mathrm{R}^{2}=0.46\right)$ y el argumento de la resonancia no parece ser contundente.

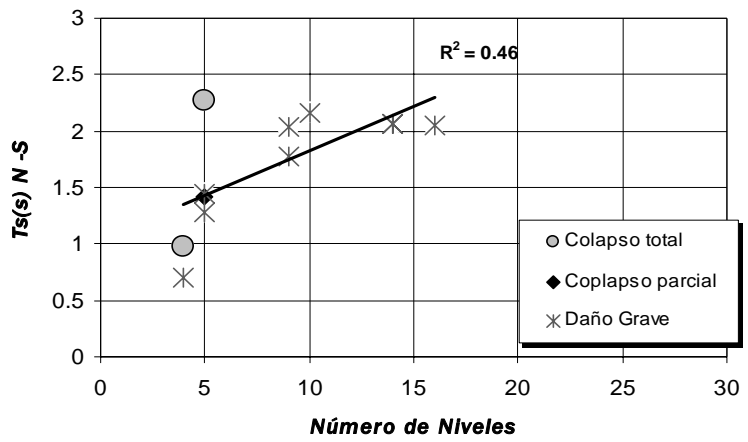

Figura 24. Correlación entre el número de niveles de las estructuras dañadas y el periodo del suelo (Ts).

En la fig. 25 se muestran las correlaciones obtenidas de Te con Ts, estimando Te con las expresiones de Muría y González (fig. 25a), Chopra y Goel (fig. 25b) y Mosquera y col. (fig. 25c). A pesar de que uno esperaría mejores resultados las correlaciones fueron muy similares a la obtenida para el número de niveles. Estas correlaciones no dejan muy en claro que la resonancia haya tenido un papel importante en las causas de daño. Se hubiera esperado que los puntos de las figs. 25 y 26 estuvieran alrededor de la línea punteada (resonancia perfecta); sin embargo, se observa una tendencia relativamente paralela en donde todos (salvo un par de ellos en el caso de la fig. 25c) los puntos caen arriba de la línea de resonancia, es decir, que el Te de las estructuras con daño siempre es menor que el del suelo hasta por un factor de dos. Esto puede explicarse por interacción suelo estructura y por comportamiento inelástico. Debido a que se sabe que tiene un efecto relativamente pequeño y a la incertidumbre que implicaría estimar la interacción de las estructuras de la tabla 1, vamos a omitir este aspecto y a enfocarnos solamente en el del comportamiento inelástico.

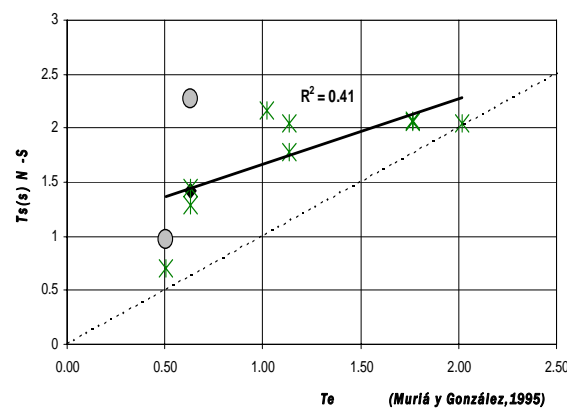

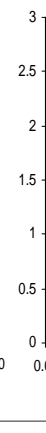

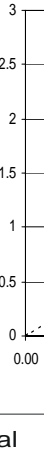

a)

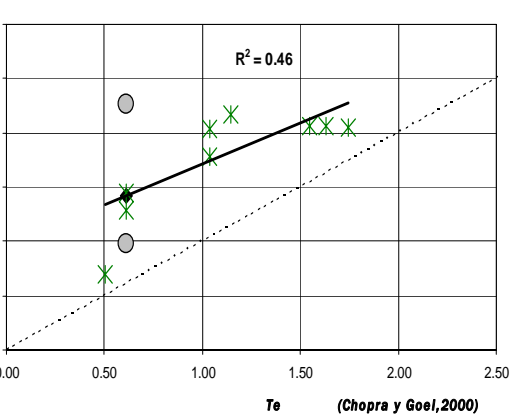

Te (Chopra y Goel, 2000)

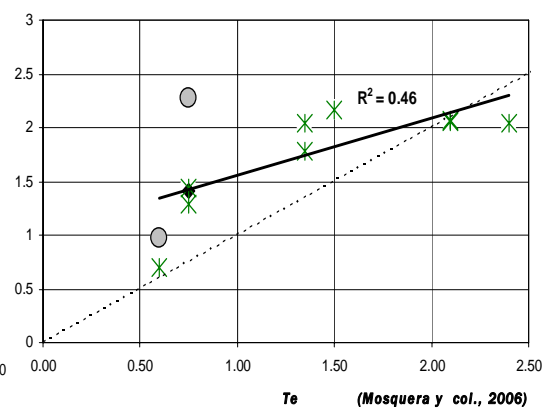

Daño Grave

b)

c)

Figura 25. Correlación entre el periodo de las estructuras dañadas (Te) por el sismo del 28/07/57 y el periodo del suelo (Ts), Te obtenido con las expresiones de a) Muría y González (1995), b) Chopra y Goel (2000) y c) Mosquera y col. (2006). Los coeficientes de correlación son bajos $\left(\mathrm{R}^{2}<0.5\right)$. 
Para considerar el posible comportamiento inelástico que pudieron haber tenido las estructuras se empleó la expresión de Newmark y Rosenblueth (1959) para las respuesta de sistemas no lineales, que predice que la modificación del periodo elástico está en función de su factor de ductilidad:

$$
\frac{T^{\prime}}{\text { To }}=\frac{1}{3 \mu}\left(1+2 \mu^{3 / 2}\right)
$$

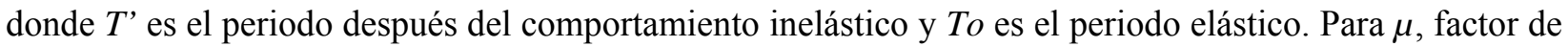
ductilidad, propusimos valores que posiblemente tenían cada una de las estructuras tomando en consideración el tipo de sistema estructural y que no se diseñaban estructuras muy dúctiles en esa época; los valores propuestos fueron: para marcos de concreto entre 1 y 1.5 , marcos de acero entre 2 y 3 , mampostería confinada y tipo nave industrial 1. En la fig. 26 se muestra la correlación obtenida entre el periodo modificado por comportamiento inelástico ( $T$, , eje horizontal) y el Ts (eje vertical), donde se aprecia que la correlación no se modifica significativamente (comparar con la fig. 25a). En la fig. 26 se indica la posición de la estructura 57-Fron de la cual se encontraron testimonios de diferentes personas afirmando que los materiales con que estaba hecha eran de mala calidad (fig. 4b); si este edificio no se tomará en cuenta, la correlación mejora bastante $\left(\mathrm{R}^{2}=0.71\right)$; lo mismo sucede para las correlaciones de la fig. 25. Sin embargo, esta información carece de bases técnicas; por lo que sólo queda como observación sobre la calidad de las construcciones y su influencia en los análisis de factores de daño.

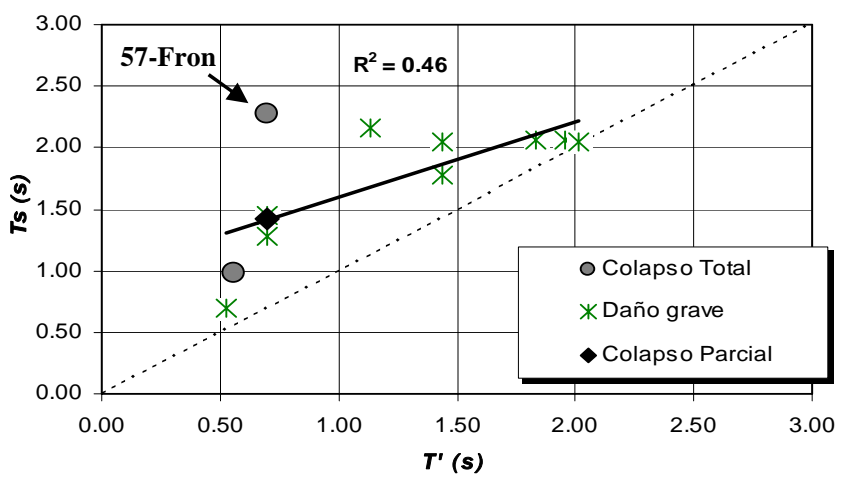

Figura 26. Correlación entre el periodo de las estructuras dañadas considerando comportamiento inelástico $\left(T^{\prime}\right)$ y el periodo del suelo (Ts), la correlación no se modifica mucho ya que los factores de ductilidad no eran muy elevados $\left(R^{2}=0.46\right)$.

Se añadieron a la correlación del Te y del Ts varias estructuras que no sufrieron daño estructural durante el sismo de 1957 y de las cuales se tiene información en el SIG. En la fig. 27 se muestra el comportamiento obtenido, la franja sombreada encierra la zona posible de resonancia tomando en cuenta que el cálculo de Te no es muy exacto. Se esperaría que dentro de esta zona estuvieran las estructuras con daños graves y fuera de ella las estructuras que no presentaron daños. Sin embargo, varias estructuras que no presentaron daño caen dentro de la franja sombreada y varias estructuras con daño están fuera de la misma. Esto quiere decir que el principal factor de daño es la resistencia (cualquiera que ésta sea) y no la resonancia. Se observa que las estructuras con daño están dentro de las líneas que indican los cocientes de $0.4<\mathrm{Te} / \mathrm{Ts} \leq 1.0$, omitiendo el caso de la estructura de 57 -Front $(\mathrm{Te} / \mathrm{Ts}=0.27)$ por lo ya expuesto es muy probable que no haya alcanzado su resistencia mucho antes de la resonancia, y que junto con las estructuras de la zona sombreada de la fig. 23 no se debería tomar en cuenta en este cálculo. 
En la fig. 28 se muestran las frecuencias de la relación Te/Ts para las estructuras existentes en 1957 de las que se tiene información en el SIG (fig. 28a) y para las que presentaron magnitudes altas de daño (fig. 28b). Se observa que para $0.4<\mathrm{Te} / \mathrm{Ts}<0.5$ están la tercera parte de las estructuras con magnitudes altas de daño que es un valor diferente al observado en las estructuras existentes; solo se tiene un caso con $\mathrm{Te} / \mathrm{Ts}<0.4$ (57-Front) y ningún caso para Te/Ts $>1$. En la fig. $28 \mathrm{~b}$ también se muestra una tabla que indica el porcentaje de las estructuras dañadas con respecto a las existentes que tenían determinado Te/Ts. Se aprecia que el máximo valor se alcanza para $\mathrm{Te} / \mathrm{Ts}=0.9$ con un 2.17 por ciento, es decir, que de las estructuras existentes las que más se dañaron fueron las que su periodo estructural $(\mathrm{Te})$ era ligeramente menor ( 0.9 veces) al del suelo $(\mathrm{Ts})$.

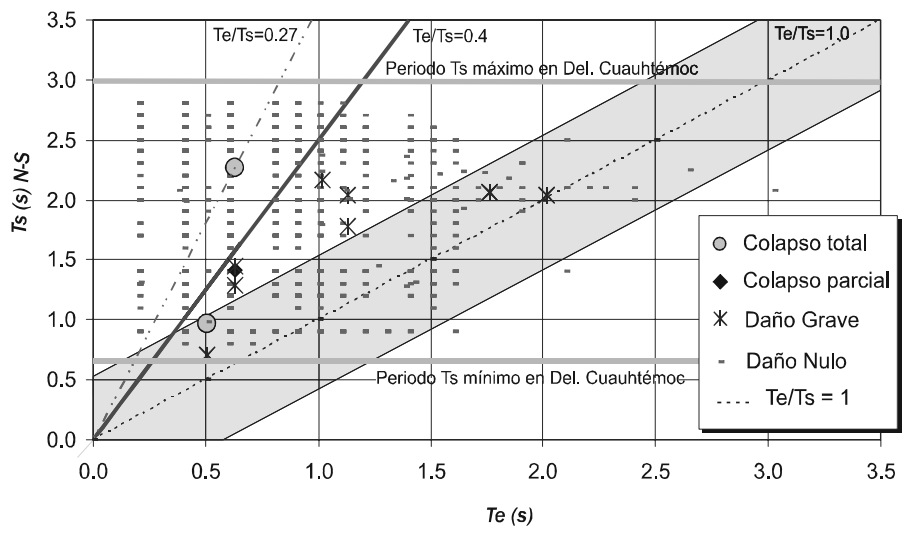

Figura 27. Correlación entre el periodo de las estructuras (Te) con y sin daño durante el sismo del 28/07/57 y el periodo del suelo (Ts). Se esperaría que las edificaciones sin daño estuvieran fuera de la franja sombreada.

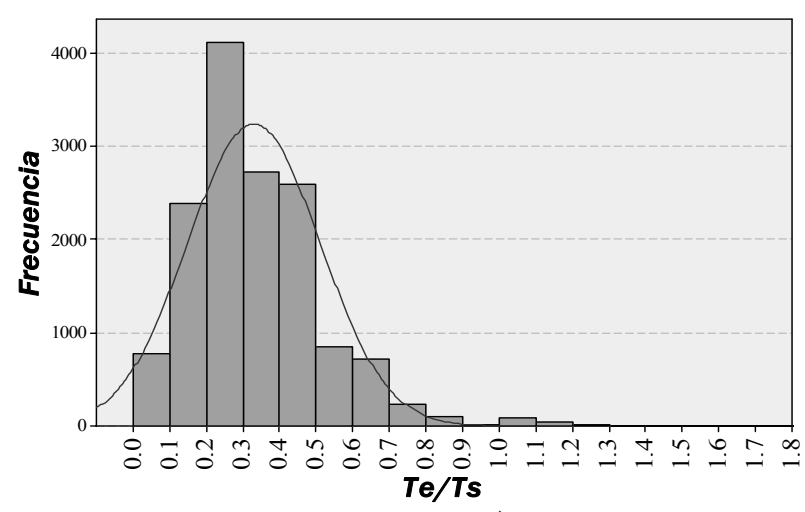

a)

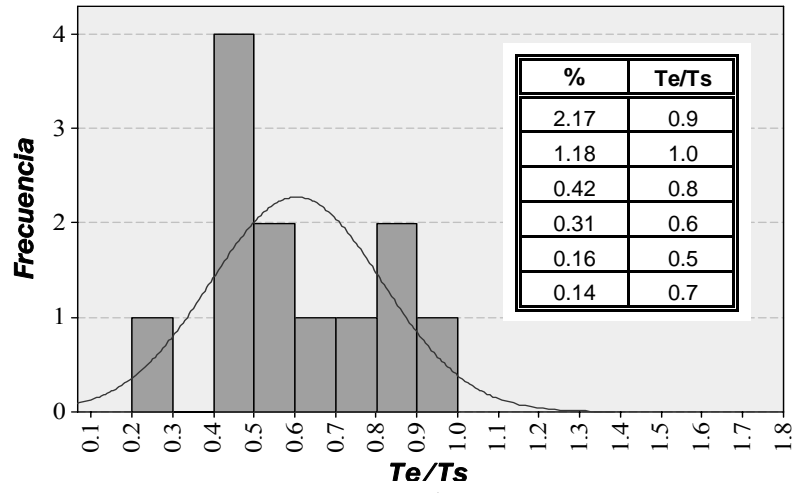

b)

Figura 28. Frecuencia de la relación Te/Ts para (a) estructuras existentes en 1957 y (b) para estructuras dañadas.

\section{CONCLUSIONES}

Se revisaron varios efectos y características de las estructuras reportadas con daño durante el sismo del 28 de julio de 1957, con el fin de identificar cuáles fueron los que más influyeron en el comportamiento sísmico de las mismas. Muchas estructuras de esa época simplemente no tuvieron un diseño sísmico y algunas de las que si lo tuvieron eran deficientes por la falta de algunos conocimientos 
que han ido surgiendo con experiencias e investigaciones recientes. A pesar de esto, es importante aclarar que es posible aprender de las estructuras colapsadas y aun más de las que no colapsaron. De lo observado en este trabajo podemos decir que el número de niveles de la estructura mostró ser un factor importante en la presencia de los daños; para este sismo en especial las estructuras de 1 a 5 niveles presentaron todos los colapsos reportados y una razón es que en el reglamento de construcción de esa época no se exigía un diseño sísmico de las edificaciones de baja altura $(16 \mathrm{~m})$ por lo que muchas de ellas no tuvieron una construcción y supervisión adecuadas. En el intervalo de estructuras con 7 a 16 niveles fue donde se presentaron la mayoría de los daños, coincidiendo con lo sucedido en sismos posteriores. Además, el número de niveles de la estructura influía en la elección del tipo de sistema estructural a utilizar que mostró ser también factor importante en la presencia de daño ya que la calidad en el diseño y construcción que se tenía en la época para cada tipo de sistema variaba mucho; actualmente se tiene más control y existen normas específicas para cada tipo. Las estructuras de concreto fueron las más dañadas seguidas por las naves industriales. Las estructuras de acero mostraron buen comportamiento y los casos reportados con daño fueron mínimos, ya que sí había bastantes estructuras de acero a pesar de que para edificios de poca altura casi no se utilizaba. Las estructuras de acero recubiertas con concreto, en esté articulo llamadas Construcción Compuesta, mostraron buen comportamiento a pesar de que no tenían el diseño de los dos materiales como se hace en la actualidad. El golpeteo entre edificios adyacentes se presentó en muy pocos casos por lo que no fue factor importante en la presencia de daños, aunque la probabilidad de que se presentara era poca por las características de las estructuras de esa época.

La ubicación en esquina mostró tener gran influencia en la presencia de daño, dos de cada cinco (40\%) de las estructuras de la tabla 1 cumplían con esto. La irregularidad en planta mostró ser también factor influyente en la presencia de daño, la mitad de las estructuras de la tabla 1 presentaban irregularidad en planta, aunque en este caso las magnitudes de daños no eran tan altas. Resalta que al tener estas dos características presentes en una estructura se convierten en un factor trascendental en la presencia de daños, más de la mitad de las estructuras en esquina tenía una gran irregularidad en planta y en su mayoría presentaron daño grave.

El tipo de cimentación fue un factor importante en la presencia de daños teniendo por ejemplo la cimentación superficial donde se reportaron todas las estructuras de colapso total y parcial; las estructuras con cimentación superficial o con pilotes de madera fueron las que más se reportaron con daños estructurales graves. Además, se observó una relación en la presencia de los asentamientos superficiales después del sismo con el tipo de cimentación y con la magnitud del daño en la estructura. Los asentamientos se presentaron en un $40 \%$ de las estructuras de la tabla 1 y estos casos se distribuyeron de forma constante en las distintas magnitudes de daño; los asentamientos se presentaron en todos los tipos de cimentación, sin embargo, las estructuras con pilotes de madera o cimentación superficial que tuvieron asentamientos fueron en su mayoría las que se reportaron con magnitudes de daño grave y colapsos.

Con la ayuda de un SIG es posible el manejo de mucha información como son la ubicación geográficamente referenciada de las estructuras, las características de las mismas, fotografías, periodo dominante del suelo, parámetros de intensidad sísmica de varios sitios para un determinado evento, entre otros, con la ventaja de poder disponer de esta información en forma visual por medio de varios mapas según se requiera. Con esta herramienta se realizaron mapas de intensidad con la intensión de encontrar alguna relación entre aceleración y el daño, observando que entre 80 y 240 gal de aceleración se presentaron los daños en las estructuras; la correlación entre el daño y las ordenadas de los espectros de respuesta fue baja. Se obtuvieron los desplazamientos y con estos las distorsiones de entrepiso, pero tampoco se encontró relación entre la magnitud de daño y los valores de las distorsiones.

A pesar de existir incertidumbre al evaluar el Te de las estructuras utilizando expresiones disponibles en la literatura, se esperaría poder observar alguna relación del efecto de resonancia con los 
daños estructurales; sin embargo, los resultados no muestran a la resonancia como factor determinante en el daño estructural. Lo que se pudo observar es que las estructuras dañadas estaban ubicadas en zonas donde el cociente de los periodos cumplía que $0.4<\mathrm{Te} / \mathrm{Ts} \leq 1.0$. Además, se compararon varias estructuras que soportaron el sismo con daño nulo y algunas de ellas estaban en zona de supuesta resonancia. Sin embargo, se encontró que de las estructuras existentes las que más daño tuvieron (2.17 por ciento del total) fueron las que presentaron un cociente $\mathrm{Te} / \mathrm{T} s \leq 0.9$.

Considerando que la excelente calidad de la información recabada en este trabajo de los edificios que sufrieron daño durante el sismo del 1957 y después de analizar las causas de este daño podemos concluir que la resonancia no es un factor que explique contundentemente estos daños, sino más bien errores de concepción estructural como irregularidades en planta y elevación, densidades de muros bajas, cimentaciones deficientes y en casos aislados, por evidentes razones del poco conocimiento de la época, baja resistencia lateral.

\section{AGRADECIMIENTOS}

Los autores agradecen la labor del M en I Carlos Eduardo Quiroga Cuéllar en varias de las actividades que dieron resultados mostrados en este trabajo. El primer autor agradece a CONACYT por la beca que le otorgó para realizar sus estudios de maestría en la Universidad Nacional Autónoma de México. Al Ing. Santiago Loera Pizarro por su grata y valiosa participación en los testimonios compartidos de los daños más relevantes por el sismo de 1957. Al Dr. Oscar González Cuevas y al M en C Enrique Del Valle por sus comentarios y la revisión crítica del trabajo, y Dr. Carlos M. Valdés González por su amable atención y ayuda para revisar los registros originales del sismo de 1957 y por facilitar varias de las fotografías originales aquí mostradas de los daños ocasionados por el sismo.

\section{REFERENCIAS}

Aguirre, M (2003), "La columna de la Independencia", "Paseo de la Reforma”, México Mágico, México, http://www.mexicomaxico.org

Bitrán, D (2001), "Características del impacto socioeconómico de los principales desastres ocurridos en México en el periodo 1980- 1999”, Serie: Impacto socioeconómico de los desastres en México, Centro Nacional de Prevención de Desastres (CENAPRED)-Secretaría de Gobernación, pp. 44-45.

Chopra, A y R Goel (2000), "Building period formulas for estimating seismic displacements”, Earthquake Spectra, Vol. 16, No.. 2, pp. 533-536.

Excelsior, El periódico de la vida nacional, Publicaciones de 29 de julio al 5 de Agosto de 1957, México, Año XLI, tomo IV.

Figueroa, J (1957), "El macrosismo del 28 de julio de 1957”, Anales del Instituto de Geofísica, UNAM, Vol. III, pp. 55-88

ICA (1985), “Experiencias derivadas de los Sismos de Septiembre de 1985”, Editorial LIMUSA y Fundación ICA. D.F., México

ICA (1992), “Catálogo de temblores que han afectado al valle de México del siglo XIV al XX”, Editorial LIMUSA y Fundación ICA. D.F., México

Marsal, R. (1958), "Los efectos del terremoto del 28 de julio y la consiguiente revisión de los criterios para el diseño sísmico de estructuras. Parte II Efectos del macrosismo registrado el 28 de julio en 
las construcciones de la ciudad”, Revista Ingeniería, Instituto de Ingeniería, UNAM, Vol. 28, No. 1, pp. 10-23.

Meli, R, O López y E Miranda (1985), "Evaluación de los efectos de los sismos de Septiembre de 1985 en los edificios de la ciudad de México Parte III. Comportamiento de edificios dañados. Anexo 1. Edificios dañados en 1957”, Instituto de Ingeniería, UNAM.

Merino, J (1957), "El temblor del 28 de julio de 1957”, Anales del Instituto de Geofísica, UNAM, Vol. III, pp. 89-125.

Miranda, E (1999), "Aproximate seismic lateral deformation demands in multistory buildings", ASCE Journal of Structural Engineering, Vol. 125, No. 4, pp. 417-425.

Mosquera, J, E Miranda y E Reinoso (2006), "Estimación y variación del periodo fundamental de vibración de edificios de concreto reforzado inferidas a partir de edificios instrumentados ubicados en la ciudad de México y California", Memorias, XV Congreso Nacional de Ingeniería Estructural Puerto Vallarta, Jal., CDROM.

Muriá, D y R González (1995), “Propiedades dinámicas de edificios de la Ciudad de México”, Revista de Ingeniería Sísmica, SMIS, No. 51, pp. 24-45.

Quiroga, C y E Reinoso (2004), "Evaluación de los daños del sismo del 19 de septiembre de 1985 a la luz de nuevos datos y estudios y con ayuda de sistemas de información geográfica", Memorias, XIV Congreso Nacional de Ingeniería Estructural Acapulco, Gro., CDROM.

Ordaz, M, L E Pérez Rocha, E Reinoso y C Montoya (1997). "Sistema de cómputo para el cálculo de espectros esperados en la Ciudad de México" Memorias, XI Congreso Nacional de Ingeniería Sísmica, Veracruz, Ver.

Orozco, V. (2006), "Estudio estadístico de efectos que influyen en el daño sísmico analizando estructuras existentes en la ciudad de México durante los sismos de 1957 y 1985 con ayuda de Sistemas de Información Geográfica" Tesis de Maestría, División de Estudios de Posgrado de la Facultad de Ingeniería, Universidad Nacional Autónoma de México.

Rosenblueth E (1958), "Los efectos del terremoto del 28 de julio y la consiguiente revisión de los criterios para el diseño sísmico de estructuras. Parte I Características de los sismos", Revista Ingeniería, Instituto de Ingeniería, UNAM, Vol. 28, No. 1, pp. 1-9.

Rosenblueth, E y N M Newmark (1976), Fundamentos de ingeniería sísmica, Editorial Diana, D.F., México, pp. 349-394.

Zeevaert, L (1960), "Base Shear on tall buildings during earthquake July 28th, 1957 in Mexico City", 2WCEE, Tokio, Japón. 